\title{
Monotonicidade em testes de hipóteses
}

\author{
Gustavo Miranda da Silva
}

\author{
DisSERTAÇÃO APRESENTADA \\ $\mathrm{AO}$ \\ Instituto DE Matemática e Estatística \\ DA \\ Universidade De SÃo PaUlo \\ PARA \\ OBTENÇÃO DO TÍTULO \\ $\mathrm{DE}$ \\ Mestre em CiênCIAS
}

Programa: Estatística

Orientador: Prof. Dr. Luís Gustavo Esteves

Durante o desenvolvimento deste trabalho o autor recebeu auxílio financeiro da CAPES/CNPq

São Paulo, março de 2010 


\section{Monotonicidade em testes de hipóteses}

Este exemplar corresponde à redação

final da dissertação/tese devidamente corrigida

e defendida por Gustavo Miranda da Silva

e aprovada pela Comissão Julgadora.

Banca Examinadora:

- Prof. Dr. Luís Gustavo Esteves (orientador) - IME-USP.

- Prof. Dr. Carlos Alberto Bragança Pereira - IME-USP.

- Prof. Dr. Verónica Andrea González-López - IMECC-UNICAMP. 


\section{Agradecimentos}

Agradeço a minha família, namorada e amigos que colaboraram direta ou indiretamente para a elaboração deste trabalho. Ao Professor Carlinhos pela suas dicas e conselhos sempre precisos. E principalmente ao Professor Luís Gustavo Esteves pela inestimável dedicação e paciência durante estes quase dois anos de colaboração.

Também a Deus, por ter jogado os seus dados para que tudo isso fosse possível. 


\section{Resumo}

A maioria dos textos na literatura de testes de hipóteses trata de critérios de otimalidade para um determinado problema de decisão. No entanto, existem, em menor quantidade, alguns textos sobre os problemas de se realizar testes de hipóteses simultâneos e sobre a concordância lógica de suas soluções ótimas.

Algo que se espera de testes de hipóteses simultâneos é que, se uma hipótese $H$ implica uma hipótese $H^{\prime}$, então é desejável que a rejeição da hipótese $H^{\prime}$ necessariamente implique na rejei-

ção da hipótese $H$, para uma mesma amostra observada. Essa propriedade é chamada aqui de monotonicidade.

A fim de estudar essa propriedade sob um ponto de vista mais geral, neste trabalho é definida a noção de classe de testes de hipóteses, que estende a função teste para uma sigma-álgebra de possíveis hipóteses nulas, e introduzida uma definição de monotonicidade.

Também é mostrado, por meio de alguns exemplos simples, que, para um nível de significância fixado, a classe de testes Razão de Verossimilhanças Generalizada (RVG) não apresenta monotonicidade, ao contrário de testes formulados sob a perspectiva bayesiana, como o teste de Bayes baseado em probabilidades a posteriori, o teste de Lindley e o FBST.

Por fim, são verificadas, sob a teoria da decisão, quando possível, quais as condições suficientes para que uma classe de testes de hipóteses tenha monotonicidade.

Palavras-chave: Classes de testes de hipóteses, Teoria da decisão, Testes de Bayes, Monotonicidade. 


\section{Abstract}

Most of the texts in the literature of hypothesis testing deal with optimality criteria for a single decision problem. However, there are, to a lesser extent, texts on the problem of simultaneous hypothesis testing and the logical consistency of the optimal solutions of such procedures.

For instance, the following property should be observed in simultaneous hypothesis testing: if a hypothesis $H$ implies a hypothesis $H^{\prime}$, then, on the basis of the same sample observation, the rejection of the hypothesis $H^{\prime}$ necessarily should imply the rejection of the hypothesis $H$. Here, this property is called monotonicity.

To investigate this property under a more general point of view, in this work, it is defined first the notion of a class of hypothesis testing, which extends the test function to a sigma-field of possible null hypotheses, and then the concept of monotonicity is introduced properly.

It is also shown, through some simple examples, that for a fixed significance level, the class of Generalized Likelihood Ratio tests (GLR) does not meet monotonicity, as opposed to tests developed under the Bayesian perspective, such as Bayes tests based on posterior probabilities, Lindley's tests and Full Bayesian Significance Tests (FBST).

Finally, sufficient conditions for a class of hypothesis testing to have monotonicity are determined, when possible, under a decision-theoretic approach.

Keywords: Class of hypothesis testing, Decision Theory, Bayes test, Monotonicity. 


\section{Sumário}

Lista de Abreviaturas $\quad$ ix

Lista de Símbolos

Lista de Tabelas $\quad$ xiii

1 Introdução $\quad 1$

1.1 Considerações Preliminares . . . . . . . . . . . . . . . . . . . 1

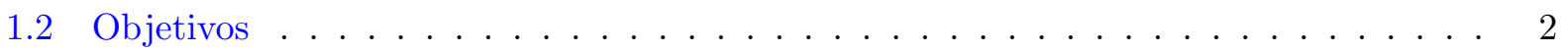

1.3 Organização do Trabalho . . . . . . . . . . . . . . . . . . 2

2 Testes de Hipóteses $\quad 3$

2.1 Testes de Hipóteses Clássicos . . . . . . . . . . . . . . . . . . . . . . . . 4

2.1.1 Teste da Razão de Verossimilhanças Generalizada . . . . . . . . . . . . . . 5

2.2 Testes de Hipóteses Bayesianos . . . . . . . . . . . . . . . . . . . . 6

2.2.1 Teste de Bayes baseados em Probabilidades a Posteriori . . . . . . . . . . . 6

2.3 FBST:Full Bayesian Significance Test . . . . . . . . . . . . . . . . 8

2.4 Teste de Lindley . . . . . . . . . . . . . . . . . . . . . . . . . . . . . . 9

3 Classes de Testes e Monotonicidade $\quad 13$

3.1 Introdução . . . . . . . . . . . . . . . . . . . . . . 13

3.2 Classes de Testes de Hipóteses e Exemplos . . . . . . . . . . . . . . . . . . . 13

3.3 Monotonicidade . . . . . . . . . . . . . . . . . . . . . 15

3.3.1 Teste de hipóteses em eleições majoritárias _ . . . . . . . . . . . . . . 16

3.3.2 Teste de hipóteses para a Distribuição Binomial . . . . . . . . . . . . . . . . . 17

3.3.3 Comparação entre as Médias de três tratamentos (Modelo ANOVA com um

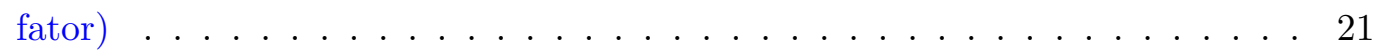

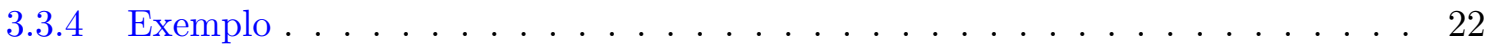

3.4 Teoria da Decisão . . . . . . . . . . . . . . . . . . . . . 23

3.4.1 Teste de Bayes . . . . . . . . . . . . . . . . . . 23

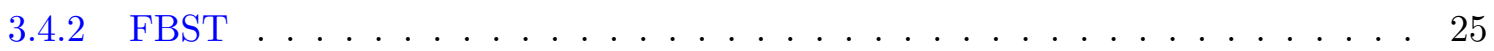

3.5 Caso Geral . . . . . . . . . . . . . . . . . . . . . . 26 
4 Conclusões

4.1 Considerações Finais . . . . . . . . . . . . . . . . . . . . 31

4.2 Sugestões para Pesquisas Futuras . . . . . . . . . . . . . . . . . . 31

A Demonstrações $\quad 33$

A.1 Construção da Região Crítica do teste RVG para o modelo de ANOVA . . . . . . . . 33

A.2 Região crítica do teste RVG para comparação entre médias de dois grupos . . . . . . 35

Referências Bibliográficas $\quad 39$ 


\title{
Lista de Abreviaturas
}

\author{
ANOVA Análise de Variância (Analysis of Variance). \\ FBST Teste de Significância Genuinamente Bayesiano (Full Bayesian Significance Test). \\ fdp Função Densidade de Probabilidade. \\ HPD Highest Posteriori Density. \\ RC Região Crítica. \\ RVG Razão de Verossimilhanças Generalizada. \\ UMP Teste Uniformemente Mais Poderoso (Uniformily Most Powerfull Test).
}




\title{
Lista de Símbolos
}

\author{
$\theta \quad$ Parâmetro. \\ $\Theta \quad$ Espaço Paramétrico. \\ $X \quad$ Vetor de observações. \\ E Espaço Amostral. \\ d Decisão. \\ $l(d, \theta)$ Função de perda associada à decisão $d$ e ao estado $\theta$. \\ $\beta \quad$ Sigma-álgebra de subconjuntos de $\theta$.
}




\section{Lista de Tabelas}

2.1 Exemplo de um Problema de Decisão . . . . . . . . . . . . . . . . . . . . . . 4

2.2 Função de perda usual para testes de hipóteses bayesianos . . . . . . . . . . . . . 7

2.3 Função de perda usual para o Full Bayesian Significance Test . . . . . . . . . . . . 8

3.1 Valores da Função $\lambda$ e da fdp da Binomial $(40 ; 0,4)$ para $H_{0} \ldots \ldots$. . . . . . . . 19

3.2 Valores da Função $\lambda$ e da fdp da Binomial $(40 ; 0,4)$ para $H_{0}^{\prime}$. . . . . . . . . . . . 20

3.3 Função de perda com valores dependendo da hipótese nula A . . . . . . . . . . 23

3.4 Função de perda para um teste FBST . . . . . . . . . . . . . . . . 25

3.5 Função de perda para uma medida finita . . . . . . . . . . . . . . . . . 27 


\section{Capítulo 1}

\section{Introdução}

\subsection{Considerações Preliminares}

O problema de teste de hipóteses, bastante recorrente na literatura estatística, é tema central no desenvolvimento de várias teorias de Inferência Estatística e ocupa papel importante na discussão entre as escolas de inferência, além da sua grande e difundida aplicação nas mais variadas áreas do conhecimento.

A grande maioria dos textos relacionados a testes de hipóteses tratam de critérios ótimos para um específico problema de decisão (teste de hipóteses), ou seja, discutem a busca de um método que, de acordo com certo critério, traga a melhor solução ao problema.

Por outro lado, existem, em menor quantidade, textos que tratam de problemas de testes de hipóteses simultâneos e a concordância lógica de suas soluções ótimas. Por exemplo, em Gabriel [9], o autor diz que "quando uma hipótese é testada por um teste de significância e não é rejeitada, é esperado que para todos os problemas de decisão com hipóteses nulas implicadas pela primeira, estas também não sejam rejeitadas, a partir do mesmo $x$ observado" (Gabriel [9]).

Alguns outros autores, além de atentarem a esse fato como Gabriel [9], também propõem algumas condições para que procedimentos de testes simultâneos para casos de Análise de Variância (ANOVA) sejam coerentes. Betz e Levin [3] mostraram que as hipóteses usuais testadas no modelo de ANOVA fatorial hierárquica (efeitos principais e interações) são conceitualmente independentes, no sentido de que a aceitação de uma hipótese independe da aceitação de outra hipótese, mesmo uma sendo subconjunto da outra, algo incoerente quando se trata deste tipo de modelo. Além disso, os autores propõem uma solução logicamente consistente para esse caso particular.

Rom e Holland [18] também apresentaram uma solução alternativa para o modelo de ANOVA fatorial hierárquico tradicional. Num contexto mais geral de problemas de decisão, Lehmann [11] mostra casos em que não se consegue chegar a uma conclusão conjunta quando se pretende responder a uma série de questionamentos em sequência e propõe uma classe de problemas de decisão múltiplos que apresenta propriedades ótimas com respeito a uma função de perda.

Em Schervish [19], é mostrado que, ao contrário do que se imagina, o p-valor para um conjunto de dados é uma função contínua da hipótese, mas não pode ser usado como medida de suporte ou evidência por apresentar incoerências lógicas (por exemplo, para o caso citado por Gabriel [9], em que uma hipótese $H$ implica uma hipótese $H^{\prime}$, mas o p-valor de $H^{\prime}$ não é pelo menos tão grande 
quanto o p-valor de $H$ ). Além disso, em Lavine e Schervish [10], os autores mostraram que o fator de Bayes também não tem coerência (no sentido mostrado por Gabriel [9]) e propõe algumas condições sobre as funções de perda de modo que os testes apresentem essa coerência.

Fossaluza [8] aborda as incoerências dos testes de hipóteses clássicos mais comuns num contexto de uma pesquisa eleitoral de uma eleição majoritária (sob modelagem multinomial) e são propostas algumas alternativas bayesianas para este caso. Mais precisamente, é citada a propriedade de monotonicidade para testes de hipóteses, algo que se espera dos procedimentos de testes simultâneos para que não ocorram alguns desses problemas de inconsistência lógica.

Este trabalho pretende verificar, para diversos testes conhecidos na literatura, quais apresentam a propriedade de monotonicidade, definida mais adiante.

De um modo mais geral, verificar-se-á, quando possível, quais as condições suficientes para que um conjunto de testes de hipóteses tenha monotonicidade.

\subsection{Objetivos}

Os objetivos deste trabalho são:

- definir classe de testes de hipóteses e apresentar alguns exemplos destas classes;

- definir a propriedade de monotonicidade e algumas de suas consequências lógicas;

- estudar classes de testes de hipóteses com relação a monotonicidade;

- definir quais as condições suficientes, sob a Teoria da Decisão, para que uma classe de testes de hipóteses tenha esta propriedade.

\subsection{Organização do Trabalho}

No Capítulo 2, apresentam-se os conceitos básicos de testes de hipóteses e são relembrados alguns testes de hipóteses mais comuns na literatura. Entre eles, o teste da Razão de Verossimilhanças Generalizada (RVG), o teste de Bayes baseado em probabilidades a posteriori, o teste de Lindley e o Full Bayesian Significance Test (FBST).

As definições formais de classe de testes de hipóteses e de monotonicidade são apresentadas no Capítulo 3. Nesse capítulo, também são estudados alguns exemplos de classes de testes de hipóteses com relação à monotonicidade, além das condições suficientes para que algumas classes de testes bayesianos tenham esta propriedade sob a abordagem da teoria da decisão.

Finalmente, no Capítulo 4 destacamos as conclusões obtidas neste trabalho. Em anexo (Apêndice A), constam algumas demonstrações de resultados auxiliares utilizados. 


\section{Capítulo 2}

\section{Testes de Hipóteses}

O principal material de uma investigação estatística é o conjunto de observações extraído de um experimento, ou seja, o valor coletado de um vetor $X$, do qual é conhecida, em geral, a menos do parâmetro $\theta$, sua distribuição $P_{\theta}$. Sobre o parâmetro $\theta$, do qual depende esta distribuição, sabe-se que ele pertence a um conjunto $\Theta$, denominado espaço paramétrico (Casella e Berger [5]).

O conjunto de todos os possíveis resultados que se pode obter da observação do vetor $X$ é chamado de espaço amostral e é representado por $\chi$. Deste modo, cada $x \in \chi$ representa uma possível amostra (realização deste vetor).

Os problemas usuais de inferência estatística consistem em usar o valor observado da amostra para obter informações a respeito da distribuição de $X$, ou sobre o parâmetro $\theta$, do qual essa distribuição depende (Lehmann [12]).

Em inferência, chamamos de hipótese estatística qualquer afirmação acerca do parâmetro, e de teste de hipóteses um problema de decisão que consiste em verificar entre duas ou mais hipóteses estatísticas, qual é mais compatível com a informação fornecida pelo experimento.

No caso mais comum, divide-se o espaço paramétrico em duas hipóteses: $H_{0}: \theta \in \Theta_{0}$ e $H_{1}: \theta \in$ $\Theta_{1}$, onde $\Theta_{1}=\Theta_{0}^{c}$, ou seja, $\left\{\Theta_{0}, \Theta_{1}\right\}$ é uma partição de $\Theta$ e denominamos $H_{0}$ e $H_{1}$ por hipótese nula e hipótese alternativa, respectivamente.

Essas hipóteses podem ser classificadas em simples, quando $\Theta_{0}$ é unitário, ou composta, caso contrário.

Elas também podem ser precisas, quando a dimensão de $\Theta_{0}\left(\operatorname{dim}\left(\Theta_{0}\right)\right)$ é menor do que a $\operatorname{dimensão~de~} \Theta(\operatorname{dim}(\Theta))$, ou não, quando $\operatorname{dim}\left(\Theta_{i}\right)=\operatorname{dim}(\Theta)$ para $i=0,1$ (Pereira e Stern [16]).

Mais formalmente,

Definição 2.1. Seja $\left\{\Theta_{0}, \Theta_{1}\right\}$ uma partição de $\Theta$. Um teste de hipóteses para $H_{0}: \theta \in \Theta_{0}$ contra $H_{1}: \theta \in \Theta_{1}$ é uma função de decisão $\varphi: \chi \rightarrow\{0,1\}$ que especifica para quais pontos $x \in \chi$ a hipótese estatística $H_{0}: \theta \in \Theta_{0}$ deve ser rejeitada ou aceita, onde $\varphi(x)=1(\varphi(x)=0)$ indica a rejeição (não-rejeição) de $H_{0}$ a partir da observação de $x$. O subconjunto do espaço amostral que nos leva à rejeição de $H_{0}$ é conhecido como região crítica $(\mathrm{RC})$ de $\varphi$, isto é, $R C=\{x \in \chi: \varphi(x)=1\}$.

Ao realizar um teste de hipóteses pode-se incorrer em dois tipos de erros. Podemos rejeitar $H_{0}$, mas $H_{0}$ estar de fato correta (erro tipo $I$ ) e podemos aceitar $H_{0}$, mas $H_{0}$ ser falsa (erro tipo $I I$ ). Essa situação é descrita na tabela 2.1. 
As diferentes abordagens ao problema de testes de hipóteses, relembradas a seguir, baseiam-se, em geral, na avaliação do risco (probabilidade) de incorrer nesses tipos de erros. A maneira como se trabalha com estes riscos leva aos diferentes procedimentos de teste de hipóteses.

\begin{tabular}{ccc}
\hline Decisão & $H_{0}$ é verdadeira & $H_{0}$ é falsa \\
\hline Aceitar $H_{0}$ & Decisão correta & Erro tipo II \\
Rejeitar $H_{0}$ & Erro tipo I & Decisão correta \\
\hline
\end{tabular}

Tabela 2.1: Possíveis consequências de um Problema de Decisão com duas hipóteses

\subsection{Testes de Hipóteses Clássicos}

Formuladas as hipóteses nula e alternativa (complementares), não é possível construir um teste de hipóteses que não apresente nenhum dos dois erros. Uma possível solução seria encontrar testes que apresentem pequenas probabilidades de cometer estes erros. No entanto, quanto menor o valor da probabilidade de cometer o erro tipo I, maior o valor da probabilidade do erro tipo II e viceversa, o que inviabiliza uma redução simultânea de tais probabilidades. Dito isso, uma maneira de encontrar um teste razoável, quando ambas as hipóteses são simples, proposta por NeymanPearson(DeGroot [6]) é: fixada a probabilidade máxima com a qual se aceita cometer o erro tipo I, encontra-se a regra de decisão que tenha a menor probabilidade do erro tipo II.

No caso de hipóteses compostas, as probabilidades de ocorrência dos erros tipo I e II, dependem, em geral, do valor de $\theta$ e são denotadas na literatura por $\alpha(\theta)$ e $\beta(\theta)$, respectivamente. Faz-se necessário, então, avaliar a seguinte função.

Definição 2.2. Considere um problema de teste de hipóteses onde $X$ é um vetor aleatório, $\theta$ é o parâmetro do qual depende a distribuição deste vetor e deseja-se testar $H_{0}: \theta \in \Theta_{0}$ contra $H_{1}: \theta \in$ $\Theta_{1}$. Seja $R C$ a região crítica do teste de hipóteses $\varphi$. A função poder de $\varphi, \pi_{\varphi}: \Theta \rightarrow[0,1]$, é dada por

$$
\pi_{\varphi}(\theta)=P(X \in R C / \theta), \text { para } \theta \in \Theta .
$$

Note que, para $\theta \in \Theta_{1}, \pi_{\varphi}(\theta)=1-\beta(\theta)$ e para $\theta \in \Theta_{0}, \pi_{\varphi}(\theta)=\alpha(\theta)$.

Como a função poder especifica, para cada valor de $\theta$, a probabilidade de $H_{0}$ ser rejeitada, o teste ideal $\varphi^{*}$ teria $\pi_{\varphi^{*}}(\theta)=1$ para todo $\theta \in \Theta_{1}$ e $\pi_{\varphi^{*}}(\theta)=0$ para todo $\theta \in \Theta_{0}$. Uma maneira de encontrar um teste, baseando-se no comentário acima, é especificar um limite superior $\alpha_{0}\left(0<\alpha_{0}<1\right)$ para o erro tipo I e, considerando somente os testes para os quais $\pi_{\varphi}(\theta) \leq \alpha_{0}$ para todo valor de $\theta \in \Theta_{0}$ (este limite superior é chamado de nível de significância do teste), maximizar $\pi_{\varphi}(\theta)$ para $\theta \in \Theta_{1}$ (DeGroot [6]).

Definindo o tamanho do teste $\varphi$ por $\alpha_{\varphi}=\sup _{\theta \in \Theta_{0}} \pi_{\varphi}(\theta)$, vale notar que, se for especificado um nível de significância $\alpha_{0}$ em um dado problema de teste de hipóteses, então serão considerados somente testes $\varphi$ com tamanho $\alpha_{\varphi} \leq \alpha_{0}$.

A partir destas idéias, segue a definição. 
Definição 2.3. Seja $C$ o conjunto de testes de hipóteses com tamanho menor ou igual a $\alpha \in(0,1)$ para testar $H_{0}: \theta \in \Theta_{0}$ contra $H_{1}: \theta \in \Theta_{1}$. Um teste $\varphi$ no conjunto $C$, com função poder $\pi_{\varphi}$, é um teste uniformemente mais poderoso (UMP) se $\pi_{\varphi}(\theta) \geq \pi_{\varphi^{\prime}}(\theta)$, para $\theta \in \Theta_{1}$ e para todo teste $\varphi^{\prime}$ na classe $C$.

Estes testes são considerados ótimos sob a perspectiva clássica e, para o caso em que ambas as hipóteses são simples, os testes UMP podem ser obtidos pelo lema de Neyman-Pearson (DeGroot [6]). Para alguns outros casos (como de distribuições amostrais pertencentes à família de distribuições monótonas [4]), ainda é possível encontrar o teste UMP. Porém, para a grande maioria dos problemas, não é fácil encontrá-los ou tais procedimentos simplesmente não existem: por exemplo, não existe teste UMP de tamanho $\alpha \in(0,1)$ para testar $H_{0}: \theta=\theta_{0}$ contra $H_{1}: \theta \neq \theta_{0}$ quando considera-se $X_{1}, X_{2}, \ldots, X_{n}$, dado $\theta$, variáveis aleatórias independentes e identicamente distribuidas $N(\theta, 1)$ (Bolfarine e Sandoval [4]).

Como alternativa, um procedimento de teste razoável e de certa forma intuitivo que pode ser utilizado em muitos casos, sem muita dificuldade, é o teste da Razão de Verossimilhanças Generalizada (RVG), relembrado a seguir.

\subsubsection{Teste da Razão de Verossimilhanças Generalizada}

Definição 2.4. Seja $X$ um vetor aleatório com função densidade de probabilidade condicional (dado $\theta)$ dada por $f(. / \theta)$ para $\theta \in \Theta$. A função de verossimilhança gerada por $x \in \chi$ é uma função $L(. / x): \Theta \rightarrow \Re_{+}$definida por:

$$
L(\theta / x)=f(x / \theta)
$$

Definição 2.5. Considere o problema de testar $H_{0}: \theta \in \Theta_{0}$ contra $H_{1}: \theta \in \Theta_{1}$ a partir de $X$ com função densidade de probabilidade condicional $f(. / \theta)$. O teste Razão de Verossimilhanças Generalizada (RVG) de tamanho $\alpha \in(0,1)$ para as hipóteses acima é definido como o teste com região crítica dada por:

$$
R C=\left\{x \in \chi: \lambda(x)=\frac{\sup _{\theta \in \Theta_{0}} L(\theta / x)}{\sup _{\theta \in \Theta} L(\theta / x)} \leq c\right\}
$$

onde c é determinado de modo que

$$
\alpha=\sup _{\theta \in \Theta_{0}} P(\lambda(X) \leq c / \theta), \alpha \in(0,1) .
$$

Observa-se que $0 \leq \lambda(x) \leq 1, \forall x \in \chi$, pois o numerador é o supremo de $L(\theta / x)$ com relação à $\theta$ pertencente a um subconjunto de $\Theta\left(\Theta_{0} \subseteq \Theta\right)$.

Os testes RVG, em geral, não são testes UMP. Porém, sob algumas condições de regularidade, apresentam boas propriedades assintóticas (Lehmann [12]). No entanto, como veremos mais adiante, quando são realizados testes de hipóteses RVG simultâneos, estes procedimentos podem apresentar incoerências lógicas. 


\subsection{Testes de Hipóteses Bayesianos}

O problema de testes de hipóteses também pode ser formulado do ponto de vista Bayesiano. Neste caso, o teste será baseado na distribuição a posteriori $\pi(. / x)$ do parâmetro $\theta$ uma vez observado o ponto amostral $x \in \chi$. A formulação bayesiana sob a perspectiva da Teoria da Decisão, na qual se define para cada possível decisão e valor de $\theta$ uma penalidade, permite não apenas a construção de regras de decisão baseadas em combinações lineares das probabilidades dos erros, mas também em outras formas de penalidades em caso de funções de perda mais gerais. Os elementos requeridos para a solução de um problema de decisão são apresentados na sequência.

\subsubsection{Teste de Bayes baseados em Probabilidades a Posteriori}

Seja $\Theta$ o espaço paramétrico (ou espaço de estados) tal que, cada $\theta \in \Theta$ representa uma possível realização do elemento desconhecido de interesse. Considere também o espaço de ações $D$, onde cada elemento $d \in D$ representa uma possível ação (ou afirmação sobre $\theta$ ) entre as quais o decisor deve escolher.

Ainda deve-se considerar uma distribuição de probabilidade a priori $\pi($.$) para \theta$ e uma função de perda $l: \Theta \times D \rightarrow R_{+}$que, para cada par $(\theta, d) \in \Theta \times D$ associa a perda $l(\theta, d)$ ocasionada pela escolha de $d$ quando $\theta$ é a realização do elemento desconhecido de interesse.

A fim de obter as informações a respeito do parâmetro $\theta$, será observado um valor $x \in \chi$ de um vetor aleatório $X$, cuja distribuição $f(. / \theta)$ depende de $\theta$.

Finalmente, uma função de decisão $\delta: \chi \rightarrow D$ especifica para cada possível valor $x \in \chi$, uma decisão $d \in D$. A classe de todas as possíveis funções de decisão será denotada por $\Delta$. A função de decisão ótima neste caso é a função $\delta^{*} \in \Delta$ que minimiza a função de perda esperada (ou risco), definida por:

$$
r(\delta)=E[l(\theta, \delta(X))]=\int_{\Theta} \int_{\chi} l(\theta, \delta(x)) f(x / \theta) \pi(\theta) d x d \theta
$$

Isto é,

$$
r\left(\delta^{*}\right)=\min _{\delta \in \Delta} r(\delta) .
$$

Minimizar esta função pode ser bastante trabalhoso. Uma forma equivalente então seria supor, para cada $x \in \chi$, que o experimento já foi realizado e que foi observado o resultado $x$. Pode-se, dessa maneira, encontrar a distribuição a posteriori para $\theta$ dado $x$. Neste caso, a decisão ótima $\delta^{*}(x)$, chamada de decisão de Bayes, é a ação $d^{*} \in D$ que minimiza a função de risco a posteriori definida por (DeGroot [7])

$$
r(d / x)=E[l(\theta, d) / x]=\int_{\Theta} l(\theta, d) \pi(\theta / x) d \theta .
$$

O problema de teste de hipóteses pode ser visto como um caso particular da teoria das decisões, onde $D=\left\{d_{0}, d_{1}\right\}$, tal que $d_{0}$ e $d_{1}$ são decisões correspondentes a decidir pela hipótese $H_{0}: \theta \in \Theta_{0}$ e decidir pela hipótese $H_{1}: \theta \in \Theta_{1}$, respectivamente, onde $\Theta_{1}=\Theta_{0}^{c}$. Ao longo deste trabalho, $d_{0}$ e $d_{1}$ são denotados (abreviados) em alguns momentos por 0 e 1, respesctivamente. 


\begin{tabular}{ccc}
\hline & $\theta \in \Theta_{0}$ & $\theta \in \Theta_{1}$ \\
\hline$d_{0}$ & 0 & $a$ \\
$d_{1}$ & $b$ & 0 \\
\hline
\end{tabular}

Tabela 2.2: Função de perda usual para testes de hipóteses bayesianos

Deste modo, a solução de Bayes para um problema de teste de hipóteses desse tipo consiste em escolher a função $\delta \in \Delta$ que minimiza a perda esperada $r($.$) , de forma que a cada x \in \chi, \delta(x)$ corresponde à decisão de rejeitar $\left(d_{1}\right)$ ou não $\left(d_{0}\right)$ a hipótese $H_{0}$. Ou seja, dado um valor $x \in \chi$, consiste em escolher a decisão $d \in\left\{d_{0}, d_{1}\right\}$ que minimiza a função de risco posterior.

Uma função de perda usual para este problema é apresentada na tabela 2.2. Baseado nessa função de perda, pode-se encontrar a decisão de Bayes para este problema de decisão.

Proposição 2.1. Um teste de Bayes para testar $H_{0}: \theta \in \Theta_{0}$ contra $H_{1}: \theta \in \Theta_{1}$ com relação à perda definida na Tabela 2.2 consiste em rejeitar $H_{0}$ (ou decidir por $d_{1}$ ), a partir de $x \in \chi$, se, e somente se,

$$
P\left(\theta \in \Theta_{0} / x\right) \leq \frac{a}{a+b}
$$

Demonstração. O risco a posteriori da decisão $d_{0}$ é:

$$
\begin{aligned}
r\left(d_{0} / x\right) & =E\left[l\left(\theta, d_{0}\right) / x\right] \\
& =\int_{\Theta} l\left(\theta, d_{0}\right) \pi(\theta / x) d \theta \\
& =\int_{\Theta_{1}} a \pi(\theta / x) d \theta \\
& =a P\left(\theta \in \Theta_{1} / x\right) \\
& =a\left[1-P\left(\theta \in \Theta_{0} / x\right)\right] .
\end{aligned}
$$

E o risco a posteriori da decisão $d_{1}$ é:

$$
\begin{aligned}
r\left(d_{1} / x\right) & =E\left[l\left(\theta, d_{1}\right) / x\right] \\
& =\int_{\Theta} l\left(\theta, d_{1}\right) \pi(\theta / x) d \theta \\
& =\int_{\Theta_{0}} b \pi(\theta / x) d \theta \\
& =b P\left(\theta \in \Theta_{0} / x\right) .
\end{aligned}
$$

Então, o procedimento de teste de Bayes consiste em rejeitar $H_{0}$ (decidir por $d_{1}$ ) se, e somente se, $r\left(d_{1} / x\right) \leq r\left(d_{0} / x\right)$, e

$$
r\left(d_{1} / x\right) \leq r\left(d_{0} / x\right) \Longleftrightarrow b P\left(\theta \in \Theta_{0} / x\right) \leq a\left[1-P\left(\theta \in \Theta_{0} / x\right)\right] \Longleftrightarrow P\left(\theta \in \Theta_{0} / x\right) \leq \frac{a}{a+b} .
$$


O teste de Bayes baseado na função de perda da tabela 2.2 é, ao longo deste trabalho, chamado de Teste de Bayes baseado em probabilidades a posteriori.

A seguir, é revisada outra abordagem para o problema de teste de hipóteses sob a perspectiva bayesiana.

\subsection{FBST:Full Bayesian Significance Test}

Uma outra abordagem bayesiana para o problema de teste de hipóteses foi proposta por Pereira e Stern [16], que definiram uma medida de evidência (e-valor) para o caso de um teste de hipóteses com hipótese nula precisa, ou seja, com probabilidade a posteriori nula (Stern [20]). Este teste apresenta uma série de vantagens em relação aos outros testes desenvolvidos para hipóteses precisas (Berger e Delampady [1]), vantagens que são descritas em Pereira e Stern [16], Pereira, Stern e Wechsler [17], Madruga et al. [14] e em Stern [20].

Essa proposta é descrita a seguir como em Madruga et al. [14].

Definição 2.6. Seja $\chi$ o espaço amostral, $\Theta$ o espaço paramétrico e $\pi$ é a densidade a priori sobre $\Theta$. Seja $\Theta_{0}$ um subconjunto de $\Theta$ que tenha medida de Lebesgue nula. Seja $\pi(\theta / x)$ a densidade a posteriori de $\theta$, observado $x \in \chi, e T(x)=\left\{\theta: \pi(\theta / x)>\sup _{\Theta_{0}}[\pi(\theta / x)]\right\}$. $A$ medida de evidência de Pereira-Stern em favor de $H_{0}$ é definida por $E V\left(\Theta_{0}, x\right)=1-P[\theta \in T(x) / x] e$ $o$ teste de Pereira-Stern, ou Full Bayesian Significance Test (FBST), consiste em aceitar $H_{0}: \Theta \in \Theta_{0}$ quando $E V\left(\Theta_{0}, x\right)$ é suficientemente "grande". Quando $\Theta_{0}=\varnothing$, convenciona-se que $\sup _{\Theta_{0}}[\pi(\theta / x)]=0$.

Este procedimento também pode ser visto como um teste de Bayes a partir da teoria da decisão, onde $D=\left\{d_{0}, d_{1}\right\}$ e $d_{0}$ e $d_{1}$ correspondem a aceitar e rejeitar $H_{0}$, respectivamente. Uma das funções de perda propostas por Madruga et al [14] é apresentada a seguir.

Seja $l: \Theta \times D$ definida por:

\begin{tabular}{ccc}
\hline & $\theta \in T(x)$ & $\theta \notin T(x)$ \\
\hline$d_{0}$ & $b+c$ & $b$ \\
$d_{1}$ & 0 & $a$ \\
\hline
\end{tabular}

Tabela 2.3: Função de perda usual para o Full Bayesian Significance Test

$\operatorname{com} a, b, c>0$.

De acordo com Madruga et al. [14], o fato da função de perda depender da observação $x$ permite incorporar alguns aspectos psicológicos de uma preferência individual. Outro aspecto dessa função de perda é que ela pune severamente o decisor que aceitar $H_{0}$, quando $\theta$ é de fato mais "provável" que a moda da posteriori sob $H_{0}$, isto é, quando $\theta \in T(x)$ (Madruga et al. [14]).

Proposição 2.2. Um teste de Bayes para testar $H_{0}: \theta \in \Theta_{0}$ contra $H_{1}: \theta \notin \Theta_{0}$ com relação à perda definida pela tabela 2.3 consiste em aceitar $H_{0}$ (ou decidir por $d_{0}$ ), a partir de $x \in \chi$, se, e somente se,

$$
E V\left(\Theta_{0}, x\right)>\frac{b+c}{a+c}
$$


Demonstração. O risco de $d_{0}$ é

$$
\begin{aligned}
E\left[l\left(\theta, d_{0}\right) / x\right] & =\int_{\Theta}[b+c I(\theta \in T(x))] \pi(\theta / x) d \theta \\
& =\int_{\Theta} b \pi(\theta / x) d \theta+\int_{T(x)} c \pi(\theta / x) d \theta \\
& =b+c\left[1-E V\left(\Theta_{0}, x\right)\right] .
\end{aligned}
$$

E o risco de $d_{1}$ é

$$
\begin{aligned}
E\left[l\left(\theta, d_{1}\right) / x\right] & =\int_{\Theta} a[1-I(\theta \in T(x))] \pi(\theta / x) d \theta \\
& =\int_{\Theta} a \pi(\theta / x) d \theta+\int_{T(x)} a \pi(\theta / x) d \theta \\
& =a E V\left(\Theta_{0}, x\right) .
\end{aligned}
$$

Então, observado $x \in \chi$, um procedimento de teste de Bayes consiste em aceitar $H_{0}$ (decidir por $\left.d_{0}\right)$ se, e somente se, $E\left[l\left(\theta, d_{0}\right) / x\right]<E\left[l\left(\theta, d_{1}\right) / x\right]$, isto é,

$$
b+c\left[1-E V\left(\Theta_{0}, x\right)\right]<a E V\left(\Theta_{0}, x\right) \Longleftrightarrow E V\left(\Theta_{0}, x\right)>\frac{b+c}{a+c} .
$$

Da proposição 2.2, nota-se que se $a<b$, então a decisão será sempre por rejeitar $H_{0}$, pois $E V\left(\Theta_{0}, x\right) \in[0,1]$. Neste caso, independente do valor de $\theta$, a perda associada à decisão $d_{1}$ é sempre menor que a perda associada a $d_{0}$.

\subsection{Teste de Lindley}

Um terceiro procedimento de teste sob a perspectiva bayesiana foi proposto por Lindley [13]. Este teste é baseado em regiões de credibilidade Highest Posteriori Density (HPD) e, segundo Lindley [13], é apropriado somente para problemas em que a informação a priori é vaga ou difusa (ou seja, quando se testar a hipótese nula $H_{0}: \theta=\theta_{0}$ contra $H_{1}: \theta \neq \theta_{0}$, e não houver razão, a priori, para acreditar que $\theta=\theta_{0}$ é mais provável que $\theta=\theta_{1}$, onde $\theta_{1}$ é algum valor de $\theta$ próximo a $\left.\theta_{0}\right)$.

Para a maioria dos casos, o procedimento de teste de Lindley leva a conclusões computacionalmente equivalentes aos testes clássicos (Zellner [21]). Entretanto, a interpretação do teste de Lindley é fundamentalmente diferente dos testes clássicos. Para o caso de testes simultâneos, será mostrada outra vantagem deste teste.

A seguir, será lembrada a definição de região de credibilidade, utilizada na formulação do teste de Lindley.

Definição 2.7. (Região de Credibilidade). Seja $\Theta$ o espaço paramétrico, $\pi($.$) a densidade a priori$ 
e $\pi(. / x)$ a densidade a posteriori de $\theta$ dado $X=x$. Uma região $C \subseteq \Theta$ tal que

$$
\int_{C} \pi(\theta / x) d \theta=1-\alpha
$$

onde $\alpha \in(0,1)$, é uma região de credibilidade para $\theta$, com respeito à posteriori de $\theta$ dado $x$, com credibilidade (ou probabilidade) $1-\alpha$.

Note que a definição acima poderia ser feita para a distribuição a priori de $\theta$.

É claro que existem várias regiões de credibilidade para um determinado valor de $\alpha \in(0,1)$. Porém, assim como nas regiões de confiança, as regiões de credibilidade com menor "volume" são as mais interessantes.

Uma região Highest Posteriori Density (HPD) é a "menor" região de credibilidade entre todas as regiões de mesma probabilidade existentes para uma determinada distribuição.

A seguir a definição formal de região HPD proposta por Bernardo e Smith [2].

Definição 2.8. (HPD) Seja $\Theta$ o espaço paramétrico, $\pi($.$) a densidade a priori e \pi(. / x)$ a densidade a posteriori para $\theta$. Uma região $C \subseteq \Theta$ é uma região Highest Posteriori Density (HPD) para $\theta$, com respeito à posteriori $\pi(. / x)$, com credibilidade $1-\alpha$, onde $\alpha \in(0,1)$, se

- $\int_{C} \pi(\theta / x) d \theta=1-\alpha e$

- $\pi\left(\theta_{1} / x\right) \geq \pi\left(\theta_{2} / x\right), \forall \theta_{1} \in C$ e $\forall \theta_{2} \in C^{c}$, exceto em algum subconjunto de $\Theta$ de probabilidade zero.

Se $\Theta \subseteq \mathbb{R}$ e a densidade a posteriori é unimodal, essas regiões correspondem ao que chamamos de intervalos de credibilidade HPD.

A idéia por trás do teste de Lindley (e de todos os testes bayesianos apresentados neste trabalho) é que a distribuição a posteriori de um parâmetro $\theta$ fornece a informação para fazer afirmações sobre o parâmetro. No caso do teste de Lindley, se o valor especificado pela hipótese nula está em uma região onde a densidade a posteriori não é alta, então este valor não é um valor muito "provável" para $\theta$ e, portanto, rejeita-se a hipótese nula.

Mais formalmente,

Definição 2.9. Seja $\Theta \subseteq \mathbb{R}, \pi(. / x)$ a densidade a posteriori para $\theta$ e $H P D_{x}(\alpha)$ um intervalo HPD para $\theta$ com respeito à posteriori $\pi(. / x)$ com credibilidade $1-\alpha$. Um teste de Lindley para testar $H_{0}: \theta=\theta_{0}$ contra $H_{1}: \theta \neq \theta_{0}$, para $\theta_{0} \in \Theta$, consiste em aceitar $H_{0}$ se, e somente se,

$$
\theta_{0} \in H P D_{x}(\alpha) \text { ou }\left\{\theta_{0}\right\} \cap H P D_{x}(\alpha) \neq \varnothing .
$$

Segundo Lindley [13], este teste deve ser usado quando a densidade a posteriori $\pi(. / x)$ é unimodal e quando a densidade a priori é vaga ou difusa.

Uma possível generalização para hipóteses compostas é apresentada abaixo. 
Definição 2.10. Seja $\Theta \subseteq \mathbb{R}^{k}, \pi(. / x)$ a densidade a posteriori para $\theta$ e $H P D_{x}(\alpha)$ uma região HPD para $\theta$ com respeito à posteriori $\pi(. / x)$ com credibilidade $1-\alpha$. Um teste de Lindley para testar as hipóteses $H_{0}: \theta \in A$ contra $H_{1}: \theta \notin A, A \subseteq \Theta$, consiste em aceitar $H_{0}$ se, e somente se,

$$
A \cap H P D_{x}(\alpha) \neq \varnothing .
$$

Para muitos problemas, o teste de Lindley leva a conclusões, aceitação ou rejeição da hipótese nula, iguais aos testes clássicos. Para amostras de tamanho grande, quando a função de verossimilhança tem distribuição assintoticamente normal com vetor de médias igual à estimativa de máxima verossimilhança do vetor $\theta$ e matriz de covariância igual ao inverso da estimativa da matriz de informação de Fisher, o procedimento de Lindley produz resultados computacionalmente equivalentes àqueles da teoria assintótica (Zellner [21]). 


\section{Capítulo 3}

\section{Classes de Testes e Monotonicidade}

\subsection{Introdução}

Muito se discute acerca do problema de teste de hipóteses. Em muitos casos, faz-se necessário considerar, simultaneamente, dois ou mais problemas de tomada de decisão a partir da mesma observação amostral. Por exemplo, suponha que se deseja testar entre $k$ tratamentos se existe alguma diferença significativa, em média, entre eles. Para isso, é comumente utilizado um modelo de Análise de Variância (ANOVA). Suponha ainda que a observação da amostra leva a aceitação da hipótese nula de igualdade entre as médias dos tratamentos. Por outro lado, poderia se testar, a partir da mesma observação, se existe diferença significativa, em média, entre dois dos tratamentos. Dado que foi aceito que não existe diferença significativa, em média, entre todos os $k$ tratamentos, espera-se que também não haja diferença significativa, em média, entre estes dois tratamentos.

Tratando de situações como esta, surge a necessidade de imposição de uma coerência lógica entre as diversas decisões a serem tomadas a partir de testes de hipóteses, caso contrário o procedimento de teste perde o seu sentido: dar uma justificativa lógico-matemática (racional) para uma tomada de decisão.

Deseja-se, assim, que os procedimentos de teste, independentemente do modelo que se esteja considerando, produzam conclusões corretas e coerentes.

A fim de avaliar a consistência lógica de procedimentos simultâneos de teste de hipóteses, serão definidos, na sequência, a idéia de classe de regras de decisão e o conceito de monotonicidade. Então serão estudadas classes de testes de hipóteses bem conhecidas com relação à propriedade de monotonicidade.

\subsection{Classes de Testes de Hipóteses e Exemplos}

Na definição 2.1, foi visto que um teste de hipóteses é uma função de decisão $\varphi: \chi \rightarrow\{0,1\}$ que "divide" o espaço amostral entre os pontos que levam a aceitar ou a rejeitar $H_{0}$. De um modo mais geral, podemos definir uma classe de testes de hipóteses, que, em linhas gerais, consiste em um conjunto de regras de decisão simultâneas que especificam para cada $x \in \chi$, a aceitação ou rejeição de cada uma das possíveis hipóteses nulas que se possa testar.

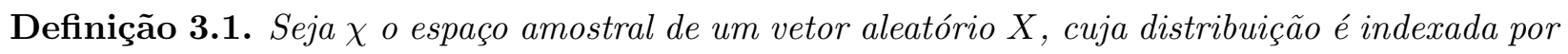
$\theta$, um parâmetro desconhecido pertencente a um espaço paramétrico $\Theta$ e $\beta$ uma sigma-álgebra de 
subconjuntos de $\Theta$. Seja $\Psi=\{\varphi: \chi \rightarrow\{0,1\}\}$ um conjunto de regras de decisão como na definição 2.1.

$A$ uma transformação $\tau: \beta \rightarrow \Psi(\tau \subseteq \beta \times \Psi)$ que a cada $A \in \beta$ associa um teste $\tau_{A} \in \Psi$ para as hipóteses $H_{0}: \theta \in A$ contra $H_{1}: \theta \notin A$, dá-se o nome de classe de testes de hipóteses.

Vale notar que, alternativamente, uma classe de procedimentos de teste poderia ser definida em outra classe de subconjuntos de $\Theta$ que não uma sigma-álgebra. Mas para acomodar probabilidades sobre $\Theta$, optou-se por essa classe de subconjuntos.

A seguir, são apresentados alguns exemplos de classes de testes de hipóteses. Para estes exemplos, são fixados o espaço paramétrico $\Theta$, a sigma-álgebra $\beta$ de subconjuntos de $\Theta$ e o espaço amostral $\chi$ de possíveis observações do vetor aleatório $X$.

Assim, para cada $A \in \beta$, podemos definir o teste $\tau_{A}$ das seguintes maneiras:

Exemplo 3.1 (Classe de Testes RVG fixado $\alpha \in(0,1))$. Uma classe $\tau$ de testes RVG para o modelo acima fixado, com nível de significância $\alpha \in(0,1)$, especifica para cada $A \in \beta$, a regra $\tau_{A}: \chi \rightarrow\{0,1\}$ para testar $H_{0}: \theta \in A$ contra $H_{1}: \theta \notin A$ dada por

$$
\tau_{A}(x)=\left\{\begin{array}{l}
0 \quad \text { se } \frac{\sup _{\theta \in A} L(\theta / x)}{\sup _{\theta \in \Theta} L(\theta / x)}>c \\
1 \quad \text { se } \frac{\sup _{\theta \in A} L(\theta / x)}{\sup _{\theta \in \Theta} L(\theta / x)} \leq c .
\end{array}\right.
$$

onde $c=c(\alpha, A)$ é determinado por $\alpha \in(0,1)$ de modo que $\sup _{\theta \in A} P\left(\tau_{A}(X)=1 / \theta\right)=\alpha$.

Exemplo 3.2 (Classe de testes de Bayes baseados em probabilidades a posteriori fixado $\alpha \in(0,1)$ ). Uma classe $\tau$ de testes de Bayes para o modelo acima fixado, com $\alpha \in(0,1)$ fixado, especifica para cada $A \in \beta$, a regra $\tau_{A}: \chi \rightarrow\{0,1\}$ para testar $H_{0}: \theta \in A$ contra $H_{1}: \theta \notin A$ dada por

$$
\tau_{A}(x)= \begin{cases}0 & \text { se } P(\theta \in A / x)>\alpha \\ 1 & \text { se } P(\theta \in A / x) \leq \alpha .\end{cases}
$$

Exemplo 3.3 (Classe de testes de Lindley fixado $\alpha \in(0,1))$. Uma classe $\tau$ de testes de Lindley para o modelo acima fixado, com $\alpha \in(0,1)$ fixado, especifica para cada $A \in \beta$, a regra $\tau_{A}: \chi \rightarrow\{0,1\}$ para testar $H_{0}: \theta \in A$ contra $H_{1}: \theta \notin A$ dada por

$$
\tau_{A}(x)= \begin{cases}0 & \text { se } A \cap H P D_{x}(\alpha) \neq \varnothing \\ 1 & \text { se } A \cap H P D_{x}(\alpha)=\varnothing\end{cases}
$$

onde $H P D_{x}(\alpha)$ é uma região HPD para $\theta$ com respeito à posteriori de $\theta$ dado $x$ de credibilidade $1-\alpha$.

Exemplo 3.4 (Classe de testes FBST). Uma classe $\tau$ de testes FBST para o modelo acima fixado, com $\alpha \in(0,1)$ fixado, especifica para cada $A \in \beta$, a regra $\tau_{A}: \chi \rightarrow\{0,1\}$, para testar $H_{0}: \theta \in A$ contra $H_{1}: \theta \notin A$, dada por 


$$
\tau_{A}(x)= \begin{cases}0 & \text { se } E V(A, x)>\alpha \\ 1 & \text { se } E V(A, x) \leq \alpha\end{cases}
$$

onde $\operatorname{EV}(A, x)$ é como na definição 2.6.

Agora será definida a propriedade de monotonicidade, que impõe uma condição lógica para tomada de decisão a partir de uma classe de testes de hipóteses.

\subsection{Monotonicidade}

Informalmente, a propriedade de "monotonicidade" em testes de hipóteses impõe que a aceitação de uma hipótese $H_{0}: \theta \in \Theta_{0}$, a partir de uma observação $x \in \chi$, implica na aceitação de uma hipótese $H_{0}^{\prime}: \theta \in \Theta_{0}^{\prime}$, a partir da mesma observação $x \in \chi$, sempre que $\Theta_{0} \subseteq \Theta_{0}^{\prime}$. A sua ausência pode implicar em incoerências como, por exemplo, uma mesma amostra levar às conclusões de que $\theta>0$ e que $\theta=0$ simultaneamente, o que não é razoável para um agente decisor racional (obediente à lógica padrão).

Essa propriedade foi estudada, com o nome de coerência, por Gabriel [9], onde ela é proposta para uma hipótese fixa ("overall") e uma família de hipóteses implicadas pela primeira, e por Schervish [19], para o caso de dois testes simultâneos. Aqui, ela é definida de um modo diferente, para uma sigma-álgebra de subconjuntos $($ de $\Theta)$ de possíveis hipóteses nulas, como pode ser visto a seguir.

Definição 3.2. Fixados o espaço amostral $\chi$, o espaço paramétrico $\Theta$, a sigma-álgebra $\beta$ de subconjuntos de $\Theta$ e seja $\tau: \beta \rightarrow \Psi$ uma classe de testes de hipóteses. A classe $\tau$ possui a propriedade de monotonicidade se $\forall A, B \in \beta$ com $A \subseteq B$ temos que $\tau_{B} \leq \tau_{A}$.

Observação 3.1. Em outras palavras, a classe $\tau$ tem monotonicidade se $\forall A, B \in \beta$ com $A \subseteq B$ vale, $\tau_{A}(x)=0$ implicar que $\tau_{B}(x)=0$. Ou ainda, se rejeitar uma hipótese $H_{0}^{\prime}: \theta \in B$ a partir de $x \in \chi$, implicar em rejeitar outra hipótese $H_{0}: \theta \in A$, a partir da mesma observação, se $A \in \beta$ é tal que $A \subseteq B$.

Veremos agora algumas classes de testes de hipóteses que apresentam esta propriedade.

Proposição 3.1. Fixados $\chi, \Theta, \beta$ e $\alpha \in(0,1)$. Seja $\tau$ a classe de testes de Bayes do exemplo 3.2. A classe $\tau$ possui a propriedade de monotonicidade.

Demonstração. Sejam $A, B \in \beta \operatorname{com} A \subseteq B$. Fixado $\alpha \in(0,1)$, sejam $\tau_{A}$ e $\tau_{B}$ duas regras de decisão definidas como no exemplo 3.2. Suponha que $\tau_{A}(x)=0$, ou seja, $P(\theta \in A / x)>\alpha$.

Como $\alpha<P(\theta \in A / x) \leq P(\theta \in B / x)$, segue que $\alpha<P(\theta \in B / x)$, ou seja, que $\tau_{B}(x)=0$. Pela observação 3.1, se para $\forall A, B \in \beta \operatorname{com} A \subseteq B$ temos $\tau_{A}(x)=0$ implica que $\tau_{B}(x)=0$, então a classe de testes $\tau$ possui a propriedade de monotonicidade.

Proposição 3.2. Fixados $\chi, \Theta, \beta$ e $\alpha \in(0,1)$. Seja $\tau$ a classe de testes de Lindley do exemplo 3.3. A classe $\tau$ possui a propriedade de monotonicidade. 
Demonstração. Sejam $A, B \in \beta \operatorname{com} A \subseteq B$. Fixado $\alpha \in(0,1)$, sejam $\tau_{A}$ e $\tau_{B}$ duas regras de decisão definidas como no exemplo 3.3. Suponha que $\tau_{A}(x)=0$, ou seja, $A \cap H P D_{x}(\alpha) \neq \varnothing$.

Como $A \subseteq B$, então $A \cap H P D_{x}(\alpha) \subseteq B \cap H P D_{x}(\alpha)$ e, portanto, $B \cap H P D_{x}(\alpha) \neq \varnothing$, ou seja, $\tau_{B}(x)=0$. Pela observação 3.1, a classe $\tau$ possui a propriedade de monotonicidade.

Proposição 3.3. Fixados $\chi, \Theta, \beta$ e $\alpha \in(0,1)$. Seja $\tau$ a classe de testes FBST do exemplo 3.4. A classe $\tau$ possui a propriedade de monotonicidade.

Demonstração. Sejam $A, B \in \beta \operatorname{com} A \subseteq B$. Fixado $\alpha \in(0,1)$, sejam $\tau_{A}$ e $\tau_{B}$ duas regras de decisão definidas como no exemplo 3.4. Supondo que $\tau_{A}(x)=0$, temos $E V(A, x)>\alpha$, ou ainda, $1-P\left(\theta \in T_{A}(x) / x\right)>\alpha$, onde $T_{A}(x)=\left\{\theta: \pi(\theta / x)>\sup _{\theta \in A}[\pi(\theta / x)]\right\}$.

Como $A \subseteq B$, então $\sup _{A}[\pi(\theta / x)] \leq \sup _{B}[\pi(\theta / x)]$ e, portanto, $T_{B}(x) \subseteq T_{A}(x)$. Se $T_{B}(x) \subseteq$ $T_{A}(x)$, então $1-P\left(\theta \in T_{A}(x) / x\right) \leq 1-P\left(\theta \in T_{B}(x) / x\right)$. Assim, $\alpha<E V(A, x) \leq E V(B, x)$ e, então, $\tau_{B}(x)=0$.

Novamente, pela observação 3.1, a classe de testes FBST $\tau$ tem monotonicidade.

Percebe-se que os testes Bayesianos apresentados, fixado $\alpha \in(0,1)$, apresentam monotonicidade. No entanto, há várias classes de testes sem monotonicidade. Como será mostrado a seguir, através de alguns exemplos, a classe de testes Razão de Verossimilhanças Generalizada (RVG) não apresenta esta propriedade em geral.

\subsubsection{Teste de hipóteses em eleições majoritárias}

Seja $X=\left(X_{1}, X_{2}, X_{3}\right) / \theta \sim \operatorname{Multinomial}\left(n, \theta_{1}, \theta_{2}, \theta_{3}\right)$, de modo que $\chi=\left\{\left(x_{1}, x_{2}, x_{3}\right) \in \mathbb{N}^{3}\right.$ : $\left.x_{1}+x_{2}+x_{3}=n\right\}$ e $\Theta=\left\{\left(\theta_{1}, \theta_{2}, \theta_{3}\right) \in \mathbb{R}_{+}^{3}: \theta_{1}+\theta_{2}+\theta_{3}=1\right\}$. No exemplo simplificado de uma eleição majoritária, ou seja, numa eleição que acaba no primeiro turno se um candidato obtiver maioria absoluta dos votos válidos (Fossaluza [8]), $X$ é um vetor aleatório que representa as contagens das intenções de voto de $n$ entrevistados em três candidatos e $\theta_{i}$ representa a proporção de votos válidos do i-ésimo candidato, $i=1,2,3$. Deseja-se saber, a partir destas contagens, se determinado candidato vencerá ou não a eleição no primeiro turno.

Primeiramente, deseja-se avaliar se nenhum dos candidatos conseguirá mais que $50 \%$ dos votos, ou seja, deseja-se testar $H_{0}: \theta \in A$ contra $H_{1}: \theta \notin A$, onde $A=\left\{\left(\theta_{1}, \theta_{2}, \theta_{3}\right) \in \Theta: \theta_{i} \leq 1 / 2, i=\right.$ $1,2,3\}=\cap_{i=1}^{3}\left\{0 \leq \theta_{i} \leq 1 / 2\right\}$.

Considerando também que se deseja saber se o j-ésimo candidato, $j \in\{1,2,3\}$, terá mais do que $50 \%$ dos votos válidos, será testado $H_{0}^{\prime}: \theta \in B$ contra $H_{1}^{\prime}: \theta \notin B$, onde $B=\left\{\left(\theta_{1}, \theta_{2}, \theta_{3}\right) \in \Theta\right.$ :

$\left.\theta_{j} \leq 1 / 2\right\}$. Sem perda de generalidade, será considerado adiante $j=1$. Para estas duas hipóteses nulas, é fácil ver que $A \subseteq B$.

Fossaluza [8] derivou os testes RVG para $H_{0}: \theta \in A$ contra $H_{1}: \theta \notin A$ e para $H_{0}^{\prime}: \theta \in B$ contra $H_{1}^{\prime}: \theta \notin B$. Para $n=1500$, temos os seguintes resultados: 
Resultado 3.1. Para o modelo acima proposto, a região crítica do teste $R V G$ (não aleatorizado) para testar $H_{0}: \theta \in A$ contra $H_{1}: \theta \notin A$, com nível de significância $\alpha=0,053$, é dada por

$$
R C=\left\{\left(x_{1}, x_{2}, x_{3}\right) \in \chi: \max \left\{x_{1}, x_{2}, x_{3}\right\} \geq 788\right\}
$$

Demonstração. Ver Fossaluza [8].

Resultado 3.2. Para o modelo acima proposto, a região crítica do teste $R V G$ (não aleatorizado) para testar $H_{0}^{\prime}: \theta \in B$ contra $H_{1}^{\prime}: \theta \notin B$, com nível de significância, $\alpha=0,053$ é dada por

$$
R C^{\prime}=\left\{\left(x_{1}, x_{2}, x_{3}\right) \in \chi: x_{1} \geq 782\right\}
$$

Demonstração. Ver Fossaluza [8].

Realizada a pesquisa de intenção de votos com $n=1500$ pessoas, foi observado o vetor $x=$ $(784,515,201)$.

Assim, para o vetor $x=(784,515,201)$ observado, $\tau_{A}(x)=0$ e, portanto, decide-se afirmar que nenhum dos candidatos terá mais de $50 \%$ dos votos, ou seja, é esperado que haja segundo turno. Por outro lado, a partir do mesmo vetor $x=(784,515,201)$ observado, $\tau_{B}(x)=1 \mathrm{e}$, portanto, há evidências de que o primeiro candidato terá mais do que $50 \%$ dos votos, ou seja, é esperado que não haja segundo turno, que são conclusões contraditórias.

Neste caso, como $A \subseteq B, \tau_{A}(x)=0, \tau_{B}(x)=1$ e pela definição 3.2, a classe de testes RVG não tem monotonicidade.

\subsubsection{Teste de hipóteses para a Distribuição Binomial}

Deseja-se avaliar $\theta \in[0,1]$ a partir de um determinado vetor aleatório $X=\left(X_{1}, X_{2}, \ldots, X_{40}\right)$ tal que $X_{i} / \theta \sim \operatorname{Bernolli}(\theta)$ de modo que $\sum_{i=1}^{40} X_{i} / \theta \sim \operatorname{Binomial}(40, \theta)$. Serão testados $H_{0}: \theta \in A$ contra $H_{1}: \theta \notin A$, onde $A=\{4 / 10\}$ e, em seguida, $H_{0}^{\prime}: \theta \in B$ contra $H_{1}^{\prime}: \theta \notin B$, onde $B=\left[0, \frac{4}{10}\right]$. Para estas duas hipóteses nulas, é fácil ver que $A \subseteq B$.

Resultado 3.3. Para o modelo acima proposto, a região crítica $R C$ do teste $R V G$ (não aleatorizado) com nível de significância $\alpha=7,44 \%$ para testar $H_{0}: \theta \in A$ contra $H_{1}: \theta \notin A$ é dada por

$$
R C=\left\{\left(x_{1}, x_{2}, \ldots, x_{40}\right) \in\{0,1\}^{40}: \sum_{i=1}^{40} x_{i} \leq 10 \text { ou } \sum_{i=1}^{40} x_{i} \geq 22\right\} .
$$

Demonstração. A função de verossimilhança nesse caso é dada por

$$
L(\theta / x)=\theta^{\sum_{i=1}^{40} x_{i}}(1-\theta)^{40-\sum_{i=1}^{40} x_{i}}
$$

para $\theta \in[0,1]$ 
Nas condições acima, considerando a hipótese $H_{0}: \theta \in A$, o valor (máximo) da função de verossimilhança sob $H_{0}$ é dado por

$$
L\left(\frac{4}{10} / x\right)=0,4^{\sum_{i=1}^{40} x_{i}}(1-0,4)^{40-\sum_{i=1}^{40} x_{i}} .
$$

E o máximo da função de verossimilhança sob $\Theta$ é dado por

$$
L(\bar{x} / x)=\bar{x}^{\sum_{i=1}^{40} x_{i}}(1-\bar{x})^{40-\sum_{i=1}^{40} x_{i}}
$$

Desta forma, segue que

$$
\lambda(x)=\lambda\left(\sum_{i=1}^{40} x_{i}\right)=\frac{(0,4)^{\sum_{i=1}^{40} x_{i}}(0,6)^{40-\sum_{i=1}^{40} x_{i}}}{\left(\frac{\sum_{i=1}^{40} x_{i}}{40}\right)^{\sum_{i=1}^{40} x_{i}}\left(1-\frac{\sum_{i=1}^{40} x_{i}}{40}\right)^{40-\sum_{i=1}^{40} x_{i}}} .
$$

Pela definição 2.5, rejeita-se $H_{0}$ se, e somente se, $\lambda(x) \leq c$, o que é equivalente a rejeitar $H_{0}$ se, e somente se, $\sum_{i=1}^{40} x_{i} \leq k_{1}$ ou $\sum_{i=1}^{40} x_{i} \geq k_{2}$. Avaliando a função $\lambda(x)=\lambda\left(\sum_{i=1}^{40} x_{i}\right)$ para $\sum_{i=1}^{40} x_{i}=0,1,2, \ldots, 40$, construíu-se a tabela 3.1 e o gráfico 3.1 .

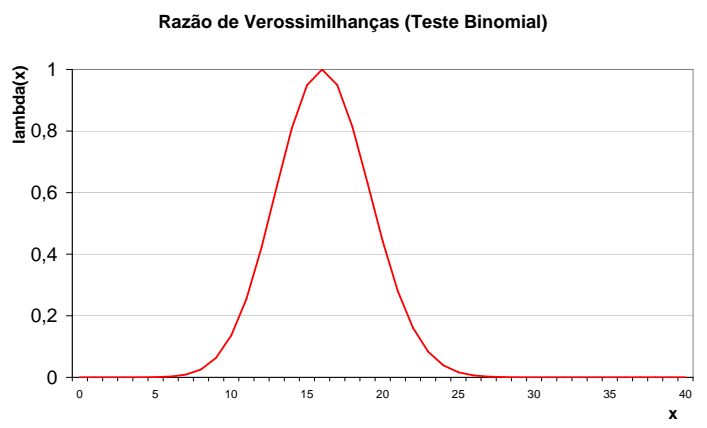

Figura 3.1: Função $\lambda(x)$ para o exemplo da Binomial.

A partir da tabela 3.1 e do gráfico 3.1, pode-se perceber que a função $\lambda(x)$ é crescente até o ponto $\sum_{i=1}^{40} x_{i}=16$ e decrescente a partir deste ponto. Portanto, o valor de $c$ tal que $P(\lambda(X) \leq$ $c / \theta=0,4)=0,0744$ é $c=0,161$, atingido em $\sum_{i=1}^{40} x_{i}=10$ e $\sum_{i=1}^{40} x_{i}=22$. Assim

$$
R C=\left\{\left(x_{1}, x_{2}, \ldots, x_{40}\right) \in\{0,1\}^{40}: \sum_{i=1}^{40} x_{i} \leq 10 \text { ou } \sum_{i=1}^{40} x_{i} \geq 22\right\} .
$$

Resultado 3.4. Para o modelo acima proposto, a região crítica $R C^{\prime}$ do teste $R V G$ (não aleatorizado) com nível de significância $\alpha=7,44 \%$ para testar $H_{0}^{\prime}: \theta \in B$ contra $H_{1}^{\prime}: \theta \notin B$ é dada 


\begin{tabular}{cccccc}
\hline$\sum x_{i}$ & $\lambda(x)$ & fdp da $\operatorname{Binomial}(40 ; 0,4)$ & $\sum x_{i}$ & $\lambda(x)$ & fdp da Binomial $(40 ; 0,4)$ \\
\hline 0 & $1,3410^{-09}$ & $1,3410^{-09}$ & 21 & $2,8010^{-01}$ & $3,5210^{-02}$ \\
1 & $9,5710^{-08}$ & $3,5610^{-08}$ & 22 & $1,6110^{-01}$ & $2,0310^{-02}$ \\
2 & $1,6710^{-06}$ & $4,6310^{-07}$ & 23 & $8,3410^{-02}$ & $1,0610^{-02}$ \\
3 & $1,6810^{-05}$ & $3,9110^{-06}$ & 24 & $3,9010^{-02}$ & $4,9910^{-03}$ \\
4 & $1,1710^{-04}$ & $2,4110^{-05}$ & 25 & $1,6510^{-02}$ & $2,1310^{-03}$ \\
5 & $6,1810^{-04}$ & $1,1610^{-04}$ & 26 & $6,2410^{-03}$ & $8,1910^{-04}$ \\
6 & $2,5910^{-03}$ & $4,5010^{-04}$ & 27 & $2,1210^{-03}$ & $2,8310^{-04}$ \\
7 & $8,9010^{-03}$ & $1,4610^{-03}$ & 28 & $6,4210^{-04}$ & $8,7610^{-05}$ \\
8 & $2,5710^{-02}$ & $4,0110^{-03}$ & 29 & $1,7310^{-04}$ & $2,4210^{-05}$ \\
9 & $6,3610^{-02}$ & $9,5110^{-03}$ & 30 & $4,0910^{-05}$ & $5,9110^{-06}$ \\
10 & $1,3610^{-01}$ & $1,9610^{-02}$ & 31 & $8,5010^{-06}$ & $1,2710^{-06}$ \\
11 & $2,5510^{-01}$ & $3,5710^{-02}$ & 32 & $1,5310^{-06}$ & $2,3810^{-07}$ \\
12 & $4,2110^{-01}$ & $5,7610^{-02}$ & 33 & $2,3510^{-07}$ & $3,8510^{-08}$ \\
13 & $6,1810^{-01}$ & $8,2710^{-02}$ & 34 & $3,0310^{-08}$ & $5,2910^{-09}$ \\
14 & $8,0910^{-01}$ & $1,0610^{-01}$ & 35 & $3,2210^{-09}$ & $6,0410^{-10}$ \\
15 & $9,4910^{-01}$ & $1,2310^{-01}$ & 36 & $2,7210^{-10}$ & $5,5910^{-11}$ \\
16 & 1 & $1,2810^{-01}$ & 37 & $1,7310^{-11}$ & $4,0310^{-12}$ \\
17 & $9,5010^{-01}$ & $1,2010^{-01}$ & 38 & $7,6410^{-13}$ & $2,1210^{-13}$ \\
18 & $8,1410^{-01}$ & $1,0310^{-01}$ & 39 & $1,9510^{-14}$ & $7,2510^{-15}$ \\
19 & $6,3110^{-01}$ & $7,9210^{-02}$ & 40 & $1,2210^{-21}$ & $1,2110^{-16}$ \\
20 & $4,4210^{-01}$ & $5,5410^{-02}$ & & & \\
\hline
\end{tabular}

Tabela 3.1: Valores da Função $\lambda$ e da fdp da $\operatorname{Binomial}(40 ; 0,4)$ para $H_{0}$

por

$$
R C^{\prime}=\left\{\left(x_{1}, x_{2}, \ldots, x_{40}\right) \in\{0,1\}^{40}: \sum_{i=1}^{40} x_{i} \geq 21\right\} .
$$

Demonstração. A função de verossimilhança neste caso é dada por

$$
L(\theta / x)=\theta^{\sum_{i=1}^{40} x_{i}}(1-\theta)^{40-\sum_{i=1}^{40} x_{i}}
$$

para $\theta \in[0,1]$

Nas condições acima, considerando a hipótese $H_{0}^{\prime}: \theta \in B$, o máximo da função de verossimilhança sob $H_{0}^{\prime}$ é dado por

$$
\max _{\theta \in B} L(\theta / x)= \begin{cases}L(\bar{x} / x)=\bar{x}^{\sum_{i=1}^{40} x_{i}}(1-\bar{x})^{40-\sum_{i=1}^{40} x_{i}}, & \text { se } \bar{x} \leq 0,4 \\ L(0,4 / x)=0,4^{\sum_{i=1}^{40} x_{i}}(1-0,4)^{40-\sum_{i=1}^{40} x_{i}}, & \text { se } \bar{x}>0,4\end{cases}
$$

E o máximo da função de verossimilhança sob $\Theta$ é dado por

$$
L(\bar{x} / x)=\bar{x}^{\sum_{i=1}^{40} x_{i}}(1-\bar{x})^{40-\sum_{i=1}^{40} x_{i}} .
$$




\begin{tabular}{cccccc}
\hline$\sum x_{i}$ & $\lambda(x)$ & fdp da $\operatorname{Binomial}(40 ; 0,4)$ & $\sum x_{i}$ & $\lambda(x)$ & fdp da Binomial $(40 ; 0,4)$ \\
\hline 0 & 1 & $1,3410^{-09}$ & 21 & $2,8010^{-01}$ & $3,5210^{-02}$ \\
1 & 1 & $3,5610^{-08}$ & 22 & $1,6110^{-01}$ & $2,0310^{-02}$ \\
2 & 1 & $4,6310^{-07}$ & 23 & $8,3410^{-02}$ & $1,0610^{-02}$ \\
3 & 1 & $3,9110^{-06}$ & 24 & $3,9010^{-02}$ & $4,9910^{-03}$ \\
4 & 1 & $2,4110^{-05}$ & 25 & $1,6510^{-02}$ & $2,1310^{-03}$ \\
5 & 1 & $1,1610^{-04}$ & 26 & $6,2410^{-03}$ & $8,1910^{-04}$ \\
6 & 1 & $4,5010^{-04}$ & 27 & $2,1210^{-03}$ & $2,8310^{-04}$ \\
7 & 1 & $1,4610^{-03}$ & 28 & $6,4210^{-04}$ & $8,7610^{-05}$ \\
8 & 1 & $4,0110^{-03}$ & 29 & $1,7310^{-04}$ & $2,4210^{-05}$ \\
9 & 1 & $9,5110^{-03}$ & 30 & $4,0910^{-05}$ & $5,9110^{-06}$ \\
10 & 1 & $1,9610^{-02}$ & 31 & $8,5010^{-06}$ & $1,2710^{-06}$ \\
11 & 1 & $3,5710^{-02}$ & 32 & $1,5310^{-06}$ & $2,3810^{-07}$ \\
12 & 1 & $5,7610^{-02}$ & 33 & $2,3510^{-07}$ & $3,8510^{-08}$ \\
13 & 1 & $8,2710^{-02}$ & 34 & $3,0310^{-08}$ & $5,2910^{-09}$ \\
14 & 1 & $1,0610^{-01}$ & 35 & $3,2210^{-09}$ & $6,0410^{-10}$ \\
15 & 1 & $1,2310^{-01}$ & 36 & $2,7210^{-10}$ & $5,5910^{-11}$ \\
16 & 1 & $1,2810^{-01}$ & 37 & $1,7310^{-11}$ & $4,0310^{-12}$ \\
17 & $9,5010^{-01}$ & $1,2010^{-01}$ & 38 & $7,6410^{-13}$ & $2,1210^{-13}$ \\
18 & $8,1410^{-01}$ & $1,0310^{-01}$ & 39 & $1,9510^{-14}$ & $7,2510^{-15}$ \\
19 & $6,3110^{-01}$ & $7,9210^{-02}$ & 40 & $1,2210^{-21}$ & $1,2110^{-16}$ \\
20 & $4,4210^{-01}$ & $5,5410^{-02}$ & & & \\
\hline
\end{tabular}

Tabela 3.2: Valores da Função $\lambda$ e da fdp da $\operatorname{Binomial}(40 ; 0,4)$ para $H_{0}^{\prime}$

Desta forma, segue que

$$
\lambda(x)=\lambda\left(\sum_{i=1}^{40} x_{i}\right)= \begin{cases}1, & \text { se } \bar{x} \leq 0,4 \\ \frac{0,4^{\sum_{i=1}^{40} x_{i}}(1-0,4)^{40-\sum_{i=1}^{40} x_{i}}}{\bar{x}^{\sum_{i=1}^{40} x_{i}}(1-\bar{x})^{40-\sum_{i=1}^{40} x_{i}}}, & \text { se } \bar{x}>0,4 .\end{cases}
$$

Pela definição 2.5, rejeita-se $H_{0}$ se, e somente se, $\lambda(x) \leq c$, o que é equivalente a rejeitar $H_{0}^{\prime}$ se, e somente se, $\sum_{i=1}^{40} x_{i} \geq k$. Avaliando a função $\lambda(x)=\lambda\left(\sum_{i=1}^{40} x_{i}\right)$ para $\sum_{i=1}^{40} x_{i}=0,1,2, \ldots, 40$ construíu-se a tabela 3.2.

A partir da tabela 3.2 , pode-se perceber que a função $\lambda(x)$ é constante até o ponto $\sum_{i=1}^{40} x_{i}=16$ e decrescente a partir deste ponto. Portanto, o valor de $c$ tal que $\sup _{\theta \in B} P(\lambda(X) \leq c / \theta)=0,0744$ é $c=0,280$, atingido em $\sum_{i=1}^{40} x_{i}=21$. Assim,

$$
R C^{\prime}=\left\{\left(x_{1}, x_{2}, \ldots, x_{40}\right) \in\{0,1\}^{40}: \sum_{i=1}^{40} x_{i} \geq 21\right\}
$$

Assim, para o vetor $x=\left(x_{1}, x_{2}, \ldots, x_{40}\right)$ tal que $\sum_{i=1}^{40} x_{i}=21$, temos, pelo resultado 3.3 , que $\tau_{A}(x)=0$, ou seja, aceita-se $H_{0}$. Por outro lado, pelo resultado $3.4, \tau_{B}(x)=1$, ou seja, rejeita-se 
$H_{0}^{\prime}$, que são conclusões contraditórias.

Como $A \subseteq B, \tau_{A}(x)=0, \tau_{B}(x)=1$ e pela definição 3.2 , a classe não tem monotonicidade.

\subsubsection{Comparação entre as Médias de três tratamentos (Modelo ANOVA com um fator)}

O modelo de ANOVA com um fator é comumente utilizado para verificar a partir de amostras observadas de $\mathrm{k}$ grupos se existem diferenças, em média, entre estes grupos ou se são todos provenientes da mesma população (Neter et al. [15]).

Deseja-se avaliar, para 3 grupos, se existem diferenças, em média, entre eles. Neste caso, $\theta=\left(\mu_{X}, \mu_{Y}, \mu_{Z}, \sigma^{2}\right)$ e $\Theta=\left\{\left(\mu_{X}, \mu_{Y}, \mu_{Z}, \sigma^{2}\right) \in \mathbb{R}^{3} \times \mathbb{R}_{+}\right\}$.

Sejam $X=\left(X_{1}, X_{2}, \ldots, X_{n}\right)$ um vetor aleatório onde $X_{i} / \theta \sim N\left(\mu_{X}, \sigma^{2}\right)$ para $i=1,2 \ldots, n$; $Y=\left(Y_{1}, Y_{2}, \ldots, Y_{n}\right)$ um vetor aleatório onde $Y_{i} / \theta \sim N\left(\mu_{Y}, \sigma^{2}\right)$ para $i=1,2 \ldots, n$; e $Z=$ $\left(Z_{1}, Z_{2}, \ldots, Z_{n}\right)$ um vetor aleatório onde $Z_{i} / \theta \sim N\left(\mu_{Z}, \sigma^{2}\right)$ para $i=1,2 \ldots, n$.

Além disso, dado $\theta$, há independência entre os três vetores $X, Y$ e $Z$, de componentes também independentes. Neste caso, $\chi=\left(\mathbb{R}^{n}\right)^{3}$.

Para avaliar a existência de diferenças entre tratamentos, será testado $H_{0}: \mu_{X}=\mu_{Y}=\mu_{Z}$ contra $H_{1}$ : c.c.. Em seguida, será testado $H_{0}^{\prime}: \mu_{X}=\mu_{Z}$ contra $H_{1}^{\prime}: \mu_{X} \neq \mu_{Z}$. Sejam $A=$ $\left\{\left(\mu_{X}, \mu_{Y}, \mu_{Z}, \sigma^{2}\right) \in \Theta: \mu_{X}=\mu_{Y}=\mu_{Z}\right\}$ e $B=\left\{\left(\mu_{X}, \mu_{Y}, \mu_{Z}, \sigma^{2}\right) \in \Theta: \mu_{X}=\mu_{Z}\right\}$ as hipóteses nulas destes dois testes. É fácil ver que $A \subseteq B$.

Resultado 3.5. Para o modelo acima proposto, a região crítica $R C$ do teste $R V G$ com $\alpha=0,05$ para testar $H_{0}: \mu_{X}=\mu_{Y}=\mu_{Z}$ contra $H_{1}$ : c.c. é dada por:

$$
R C=\left\{(x, y, z) \in \chi: \frac{Q M E}{Q M R} \geq 3,32\right\}
$$

onde

$$
\begin{gathered}
\widehat{\mu_{0}}=\frac{\sum_{i=1}^{n} x_{i}+\sum_{i=1}^{n} y_{i}+\sum_{i=1}^{n} z_{i}}{3 n}, \\
\widehat{\mu_{X}}=\bar{x}, \\
\widehat{\mu_{Y}}=\bar{y}, \\
\widehat{\mu_{Z}}=\bar{z} \\
Q M E=\frac{\sum_{i=1}^{n}\left(\widehat{\mu}_{X}-\widehat{\mu}_{0}\right)^{2}+\sum_{i=1}^{n}\left(\widehat{\mu}_{Y}-\widehat{\mu}_{0}\right)^{2}+\sum_{i=1}^{n}\left(\widehat{\mu}_{Z}-\widehat{\mu}_{0}\right)^{2}}{2} e \\
Q M R=\frac{\sum_{i=1}^{n}\left(x_{i}-\widehat{\mu}_{X}\right)^{2}+\sum_{i=1}^{n}\left(y_{i}-\widehat{\mu}_{Y}\right)^{2}+\sum_{i=1}^{n}\left(z_{i}-\widehat{\mu}_{Z}\right)^{2}}{3 n-3} .
\end{gathered}
$$

Demonstração. Apêndice A.

Resultado 3.6. Para o modelo acima proposto, a região crítica $R C^{\prime}$ do teste $R V G$ com $\alpha=0,05$ 
para testar $H_{0}^{\prime}: \mu_{X}=\mu_{Z}$ contra $H_{1}^{\prime}: \mu_{X} \neq \mu_{Z}$ é dada por:

$$
R C^{\prime}=\left\{(x, y, z) \in \chi:\left|\frac{\bar{x}-\bar{z}}{\sqrt{Q M R} \sqrt{\frac{2}{n}}}\right| \geq 2,042\right\} .
$$

Demonstração. Apêndice A.

Assim, para os vetores, $x=(0,1,2, \ldots, 10), y=(1,2,3, \ldots, 11)$ e $z=(3,4,5, \ldots, 13)$ observados, temos $\frac{Q M E}{Q M R}=2,33$ e, portanto, não há evidências que existe diferença entre as médias dos três grupos, ou seja, aceita-se $H_{0}$ e $\tau_{A}(x, y, z)=0$. Por outro lado, $\frac{\bar{x}-\bar{z}}{\sqrt{Q M R} \sqrt{\frac{2}{n}}}=-2,12$ e, portanto, há evidências que as médias de dois grupos são diferentes, ou seja, rejeita-se $H_{0}^{\prime}$ e $\tau_{B}(x, y, z)=1$, que são conclusões contraditórias.

Novamente, como $A \subseteq B, \tau_{A}(x, y, z)=0, \tau_{B}(x, y, z)=1$ e pela definição 3.2 , a classe $\tau$ também não tem monotonicidade.

A seguir, é exibida uma formulação equivalente de monotonicidade.

Proposição 3.4. Seja $\tau$ uma classe de testes de hipóteses. Essa classe possui monotonicidade se, e somente se, $\tau_{A \cup B} \leq \tau_{A} \tau_{B}, \forall A, B \in \beta$.

Demonstração. Para provar a proposição acima, basta provar que $\tau$ não possui monotonicidade se, e somente se, $\exists A, B \in \beta$ e $x \in \chi$ tal que $\tau_{A \cup B}(x)>\tau_{A}(x) \tau_{B}(x)$.

Seja $\tau$ uma classe de testes que não possui monotonicidade. Pela definição 3.2, existem $A, B \in \beta$ $\operatorname{com} A \subseteq B$ e $x \in \chi$ tal que $\tau_{B}(x)>\tau_{A}(x)$, ou seja, $\tau_{B}(x)=1$ e $\tau_{A}(x)=0$.

Como $A \subseteq B$, então $A \cup B=B$ e, portanto, $\tau_{A \cup B}(x)=\tau_{B}(x)=1$. Conclui-se que, neste caso, $\tau_{A \cup B}(x)>\tau_{A}(x) \tau_{B}(x)$.

Por outro lado, se existem $A, B$ e $x$ tal que $\tau_{A \cup B}(x)>\tau_{A}(x) \tau_{B}(x)$, segue que $\tau_{A \cup B}(x)=1$ e que ou $\tau_{B}(x)=0$ ou $\tau_{A}(x)=0$.

Suponha, sem perda de generalidade, $\tau_{A}(x)=0$. Logo, existem conjuntos $A$ e $A \cup B(A \subseteq A \cup B)$ com $\tau_{A}(x)=0$ e $\tau_{A \cup B}(x)=1$. Logo, a classe não possui monotonicidade.

A seguir, um exemplo onde há monotonicidade, porém $\tau_{A \cup B} \neq \tau_{A} \tau_{B}$.

\subsubsection{Exemplo}

Seja $X / \theta \sim \operatorname{Bernoulli}(\theta)$ e considere, a priori, $\theta \sim \operatorname{Beta}(1,1)$. Suponha que seja observado $X=1$ de forma que $\theta / X=1 \sim \operatorname{Beta}(2,1)$. Vimos que a classe de teste de Bayes que para cada $A \in \mathbf{B}([0,1])$, onde $\mathbf{B}([\mathbf{0 , 1}])$ é a sigma-álgebra de Borel de $[0,1]$, associa o teste

$$
\tau_{A}(x)= \begin{cases}0 & \text { se } P(\theta \in A / x)>1 / 2 \\ 1 & \text { se } P(\theta \in A / x) \leq 1 / 2\end{cases}
$$

para testar $H_{0}: \theta \in A$ contra $H_{1}: \theta \notin A$ possui monotonicidade. 


\begin{tabular}{ccc}
\hline & $\theta \in A$ & $\theta \notin A$ \\
\hline$d_{0}$ & 0 & $a_{A}$ \\
$d_{1}$ & $b_{A}$ & 0 \\
\hline
\end{tabular}

Tabela 3.3: Função de perda com valores dependendo da hipótese nula A

Tomando $A=[1 / 2 ; 3 / 4]$ e $B=[3 / 4 ; 1]$, verifica-se que $\tau_{A}(x)=1$ e $\tau_{B}(x)=1$, mas $\tau_{A \cup B}(x)=0$, pois $P(\theta \in A / x)=5 / 16 \leq 1 / 2, P(\theta \in B / x)=7 / 16 \leq 1 / 2$ e $P(\theta \in A \cup B / x)=3 / 4>1 / 2$.

A seguir, será estudada a propriedade de monotonicidade em classes de testes bayesianos sob a abordagem da teoria da decisão.

\subsection{Teoria da Decisão}

Fixado um valor para $\alpha \in(0,1)$, verificou-se que os testes Bayesianos baseados em probabilidades a posteriori (exemplo 3.2) apresentam a propriedade de monotonicidade. Sob o ponto de vista da Teoria da Decisão, cada teste desta classe é obtido a partir da minimização da esperança de uma função de perda da forma da tabela 2.2. Mais precisamente, para $A \in \beta$, considera-se a função de perda $l_{A}$ dada por

$$
\begin{gathered}
l_{A}\left(d_{0}, \theta\right)=a I_{A^{c}}(\theta) \mathrm{e} \\
l_{A}\left(d_{1}, \theta\right)=b I_{A}(\theta) .
\end{gathered}
$$

Assim, para a construção da classe de testes do exemplo 3.2, deve-se considerar uma classe de funções de perda $\left(l_{A}\right)_{A \in \beta}$ (uma função de perda para a derivação de cada teste da classe) e não uma única função de perda para a obtenção de todos os testes da classe.

De um modo mais geral, pode-se considerar classes de funções de perda como a da tabela 2.2 nas quais as penalidades associadas aos erros de decisão, $a$ e $b$, dependem da hipótese nula $A \in \beta$, como na tabela 3.3 .

Para os testes baseados em probabilidades a posteriori, pode-se encontrar classes de funções de perda que confiram monotonicidade à classe de testes (isto é, para cada hipótese nula $A \in \beta$ será considerada uma tabela da forma 3.3).

\subsubsection{Teste de Bayes}

Proposição 3.5. Seja $\tau$ uma classe de testes de Bayes em que, para cada $A \in \beta, \tau_{A}$ é um teste de Bayes para testar $H_{0}: \theta \in A$ contra $H_{1}: \theta \notin A$ considerando uma função de perda da forma da tabela 3.3.

Se $\forall A, B \in \beta$ tal que $A \subseteq B$ temos $\frac{a_{A}}{a_{B}} \geq \frac{b_{A}}{b_{B}}$, então a classe de testes $\tau$ terá monotonicidade.

Demonstração. Sejam $A, B \in \beta$ tal que $A \subseteq B$. Pela proposição 2.1, rejeita-se $H_{0}: \theta \in A$ se, e somente se, $P(\theta \in A / x) \leq \frac{a_{A}}{a_{A}+b_{A}}$ e rejeita-se $H_{0}^{\prime}: \theta \in B$ se, e somente se, $P(\theta \in B / x) \leq \frac{a_{B}}{a_{B}+b_{B}}$.

A monotonicidade é violada quando, ao mesmo tempo, rejeita-se $H_{0}^{\prime}$ e aceita-se $H_{0}$, ou seja,

$$
P(\theta \in B / x) \leq \frac{a_{B}}{a_{B}+b_{B}} \text { e } P(\theta \in A / x)>\frac{a_{A}}{a_{A}+b_{A}}
$$


Como $A \subseteq B$ e por (3.1), temos que:

$$
\frac{a_{A}}{a_{A}+b_{A}}<P(\theta \in A / x) \leq P(\theta \in B / x) \leq \frac{a_{B}}{a_{B}+b_{B}} .
$$

Então,

$$
\frac{a_{A}}{a_{A}+b_{A}}<\frac{a_{B}}{a_{B}+b_{B}}
$$

Isso é equivalente a

$$
a_{A} b_{B}<a_{B} b_{A}
$$

Portanto, se

$$
a_{A} b_{B} \geq a_{B} b_{A} \quad \text { ou, equivalentemente, } \quad \frac{a_{A}}{a_{B}} \geq \frac{b_{A}}{b_{B}},
$$

a classe de teste de hipóteses $\tau$ tem monotonicidade.

Vale notar que, como $A \subseteq B$, parece razoável que a perda por tomar a decisão de rejeitar $H_{0}: \theta \in A$ quando $\theta \in A\left(b_{A}\right)$ seja menor ou igual à perda por tomar a decisão de rejeitar $H_{0}^{\prime}: \theta \in B$ quando $\theta \in A\left(b_{B}\right)$. Ou seja, rejeitar a hipótese de que $\theta \in A$, quando, de fato, $\theta \in A$, intuitivamente, parece ser menos grave do que rejeitar que $\theta \in B$, quando $\theta \in A$, pois $A \subseteq B$.

O mesmo raciocínio vale considerando que $B^{C} \subseteq A^{C}$ e, então, $a_{A} \geq a_{B}$ parece razoável.

Observação 3.2. Considere novamente a classe de testes da proposição 3.5. Se $\forall A, B$ com $A \subseteq B$, $a_{A} \geq a_{B}$ e $b_{B} \geq b_{A}$, então $\tau$ terá monotonicidade.

Demonstração. Como

$$
a_{A} \geq a_{B} \text { e } b_{B} \geq b_{A} \Rightarrow \frac{a_{A}}{a_{B}} \geq 1 \geq \frac{b_{A}}{b_{B}} \Rightarrow \frac{a_{A}}{a_{B}} \geq \frac{b_{A}}{b_{B}}
$$

e, pela proposição 3.5 ,

$$
\frac{a_{A}}{a_{B}} \geq \frac{b_{A}}{b_{B}} \Rightarrow \text { Monotonicidade. }
$$

Então,

$$
a_{A} \geq a_{B} \text { e } b_{B} \geq b_{A} \Rightarrow \text { Monotonicidade. }
$$

Ou seja, considerando classes de funções de perda intuitivas, que obedecem às condições da observação 3.2, a classe de testes de Bayes baseados em probabilidades a posteriori apresenta monotonicidade, o que indica que estas relações entre as funções de perda da classe $\left(l_{A}\right)_{A \in \beta}$, bastante razoáveis à primeira vista, são coerentes para a classe de testes de Bayes no sentido de conferirem monotonicidade à classe. Em outras palavras, monotonicidade, nesse caso, pode ser vista como uma propriedade decorrente de afirmações (julgamentos) consistentes sobre os possíveis erros de tomada de decisão em testes simultâneos. 


\subsubsection{FBST}

Podem-se obter condições semelhantes para que a classe de Testes Genuinamente Bayesianos FBST possua monotonicidade.

Proposição 3.6. Seja $\tau$ uma classe de testes FBST que, para cada $A \in \beta, \tau_{A}$ é um teste FBST para testar $H_{0}: \theta \in A$ contra $H_{1}: \theta \notin A$ construído a partir de uma função de perda da forma da tabela 3.4.

Se $\forall A, B \in \beta$ tal que $A \subseteq B$ temos

$$
\frac{b_{A}+c_{A}}{a_{A}+c_{A}} \geq \frac{b_{B}+c_{B}}{a_{B}+c_{B}}
$$

então a classe de testes $\tau$ tem monotonicidade.

\begin{tabular}{ccc}
\hline & $\theta \in T_{A}(x)$ & $\theta \notin T_{A}(x)$ \\
\hline$d_{0}$ & $b_{A}+c_{A}$ & $b_{A}$ \\
$d_{1}$ & 0 & $a_{A}$ \\
\hline
\end{tabular}

Tabela 3.4: Função de perda para um teste FBST

Demonstração. Sejam $A, B \in \beta$ tal que $A \subseteq B$. Pela proposição 2.2, rejeita-se $H_{0}: \theta \in A$ se, e somente se, $E V(A, x) \leq \frac{b_{A}+c_{A}}{a_{A}+c_{A}}$ e rejeita-se $H_{0}^{\prime}: \theta \in B$ se, e somente se, $E V(B, x) \leq \frac{b_{B}+c_{B}}{a_{B}+c_{B}}$.

A monotonicidade é violada quando, ao mesmo tempo, rejeita-se $H_{0}^{\prime}$ e aceita-se $H_{0}$, ou seja,

$$
E V(B, x) \leq \frac{b_{B}+c_{B}}{a_{B}+c_{B}}
$$

e

$$
E V(A, x)>\frac{b_{A}+c_{A}}{a_{A}+c_{A}}
$$

Como

$$
T_{A}(x)=\left\{\theta \in \Theta: \pi(\theta / x)>\sup _{\theta \in A} \pi(\theta / x)\right\}
$$

e

$$
T_{B}(x)=\left\{\theta \in \Theta: \pi(\theta / x)>\sup _{\theta \in B} \pi(\theta / x)\right\},
$$

os conjuntos tangentes às hipóteses $H_{0}$ e $H_{0}^{\prime}$, respectivamente, e como $A \subseteq B$, é fácil ver que

$$
\sup _{\theta \in B}(\pi(\theta / x)) \geq \sup _{\theta \in A}(\pi(\theta / x))
$$

e

$$
T_{B}(x) \subseteq T_{A}(x)
$$


Então,

$$
P\left[\theta \in T_{A}(x) / x\right] \geq P\left[\theta \in T_{B}(x) / x\right]
$$

e segue que

$$
1-P\left[\theta \in T_{A}(x) / x\right] \leq 1-P\left[\theta \in T_{B}(x) / x\right],
$$

isto é,

$$
E V(A, x) \leq E V(B, x)
$$

De (3.2), (3.3) e (3.4), temos que:

$$
\frac{b_{A}+c_{A}}{a_{A}+c_{A}}<E V(A, x) \leq E V(B, x) \leq \frac{b_{B}+c_{B}}{a_{B}+c_{B}}
$$

e, então,

$$
\frac{b_{A}+c_{A}}{a_{A}+c_{A}}<\frac{b_{B}+c_{B}}{a_{B}+c_{B}} .
$$

Portanto, se

$$
\frac{b_{A}+c_{A}}{a_{A}+c_{A}} \geq \frac{b_{B}+c_{B}}{a_{B}+c_{B}}
$$

a classe de testes $\tau$ tem monotonicidade.

\subsection{Caso Geral}

A partir dos casos citados na seção anterior, é natural investigar quais classes de funções de perda (mais gerais) fazem com que uma classe de testes de Bayes qualquer tenha a propriedade de monotonicidade. A seguir, são exibidos alguns resultados.

Proposição 3.7. Sejam $\chi$, $\Theta$ e $\beta$ como na definição 3.1 e $D=\left\{d_{0}, d_{1}\right\}$. Para cada $A \in \beta$, seja $l_{A}: \Theta \times D \rightarrow \Re_{+}$uma função de perda que, para cada par $(\theta, d) \in \Theta \times D$ associa a perda $l_{A}(\theta, d)$ quando se testa $H_{0}: \theta \in A$ contra $H_{1}: \theta \notin A$. Assim, temos uma classe de funções de perda $\left\{l_{A}: A \in \beta\right\}$. Suponha que a classe satisfaça

$$
\begin{gathered}
\text { (i) } l_{A}\left(\theta, d_{0}\right) \geq l_{B}\left(\theta, d_{0}\right), \forall \theta \in \Theta e \forall A, B \in \beta \operatorname{com} A \subseteq B, e \\
(i i) l_{A}\left(\theta, d_{0}\right)=l_{A^{c}}\left(\theta, d_{1}\right), \forall \theta \in \Theta e \forall A \in \beta .
\end{gathered}
$$

Então, a classe de testes que para cada $A \in \beta$ associa o teste de Bayes para testar $H_{0}: \theta \in A$ contra $H_{1}: \theta \notin A$ obtido considerando a função de perda $l_{A}$, tem monotonicidade.

Demonstração. Suponha que $\tau_{A}(x)=0$, isto é, $E\left(l_{A}\left(\theta, d_{0}\right) / x\right)<E\left(l_{A}\left(\theta, d_{1}\right) / x\right)$. De (i), temos que

$$
E\left(l_{B}\left(\theta, d_{0}\right) / x\right) \leq E\left(l_{A}\left(\theta, d_{0}\right) / x\right)<E\left(l_{A}\left(\theta, d_{1}\right) / x\right) .
$$

Se $A \subseteq B$, então $B^{c} \subseteq A^{c}$, e, por (i), temos que $l_{B^{c}}\left(\theta, d_{0}\right) \geq l_{A^{c}}\left(\theta, d_{0}\right)$. Juntado com (ii), temos

$$
l_{B}\left(\theta, d_{1}\right)=l_{B^{c}}\left(\theta, d_{0}\right) \geq l_{A^{c}}\left(\theta, d_{0}\right)=l_{A}\left(\theta, d_{1}\right),
$$


ou seja, $l_{B}\left(\theta, d_{1}\right) \geq l_{A}\left(\theta, d_{1}\right)$ e, portanto,

$$
E\left(l_{B}\left(\theta, d_{1}\right) / x\right) \geq E\left(l_{A}\left(\theta, d_{1}\right) / x\right)
$$

Juntando (3.5) com (3.6), temos

$$
E\left(l_{B}\left(\theta, d_{0}\right) / x\right) \leq E\left(l_{A}\left(\theta, d_{0}\right) / x\right)<E\left(l_{A}\left(\theta, d_{1}\right) / x\right) \leq E\left(l_{B}\left(\theta, d_{1}\right) / x\right)
$$

e, portanto, $\tau_{B}(x)=0$.

Assim, a classe de testes de Bayes, sob estas condições, possui monotonicidade.

A classe de funções de perda $\left\{l_{A}: A \in \beta\right\}$ da proposição 3.7 é também razoável, pois a condição (i) exige que, ao tomar a decisão de aceitar a hipótese $\left(d_{0}\right)$, a perda ao se testar $H_{0}: \theta \in A, l_{A}\left(\theta, d_{0}\right)$, deve ser sempre maior ou igual a perda ao se testar $H_{0}^{\prime}: \theta \in B, l_{B}\left(\theta, d_{0}\right)$, pois como $A \subseteq B$, quando $\theta \in A^{c} \cap B$, é mais grave aceitar (erroneamente) $H_{0}$, do que aceitar (acertadamente) $H_{0}^{\prime}$. Nos outros casos, $d_{0}$ é a decisão correta ou errada para as duas hipóteses nulas. A igualdade em (i) vale quando $A=B$, ou quando $\theta$ pertence ao subconjunto $A$ e, consequentemente, também pertence ao subconjunto $B$.

Já a condição (ii) exige que a perda ao tomar a decisão de aceitar $H_{0}: \theta \in A$ seja igual a perda ao tomar a decisão de rejeitar $H: \theta \in A^{c}$, o que é também intuitivo.

A seguir, alguns exemplos de classes de funções de perda que atendem às condições (i) e (ii) da proposição 3.7 .

Exemplo 3.5. Considere, para cada $A \in \beta$, uma função de perda $l_{A}$ para testar $H_{0}: \theta \in A$ contra $H_{1}: \theta \in A^{c}$ dada pela tabela abaixo

\begin{tabular}{ccc}
\hline & $\theta \in A$ & $\theta \in A^{c}$ \\
\hline$d_{0}$ & 0 & $\lambda\left(A^{c}\right)$ \\
$d_{1}$ & $\lambda(A)$ & 0 \\
\hline
\end{tabular}

Tabela 3.5: Função de perda para uma medida finita

onde $\lambda$ é uma medida finita sobre $\beta$ com $\lambda(\Theta)>0$. A classe de funções de perda $\left\{l_{A}\right\}_{A \in \beta}$ obedece a (i) e (ii) da proposição 3.7.

Demonstração. Sejam $A, B \in \beta \operatorname{com} A \subseteq B$. Da definição,

$$
l_{A}\left(\theta, d_{0}\right)= \begin{cases}\lambda\left(A^{c}\right) & \text { se } \theta \in A^{c} \\ 0 & \text { se } \theta \notin A^{c}\end{cases}
$$

e

$$
l_{B}\left(\theta, d_{0}\right)= \begin{cases}\lambda\left(B^{c}\right) & \text { se } \theta \in B^{c} \\ 0 & \text { se } \theta \notin B^{c}\end{cases}
$$


Se $A \subseteq B$, então $B^{c} \subseteq A^{c}$. Para $\theta \in A^{c} \cap B^{c}=B^{c}$

$$
l_{A}\left(\theta, d_{0}\right) \geq l_{B}\left(\theta, d_{0}\right), \text { pois } \lambda\left(A^{c}\right) \geq \lambda\left(B^{c}\right)
$$

Para $\theta \in A^{c}-B^{c}$

$$
l_{A}\left(\theta, d_{0}\right) \geq l_{B}\left(\theta, d_{0}\right) \text {, pois } \lambda\left(A^{c}\right) \geq 0 .
$$

Para $\theta \in A$

$$
l_{A}\left(\theta, d_{0}\right)=l_{B}\left(\theta, d_{0}\right)=0 .
$$

Portanto, $\forall \theta \in \Theta$ vale que $l_{A}\left(\theta, d_{0}\right) \geq l_{B}\left(\theta, d_{0}\right)$.

Como

$$
l_{A}\left(\theta, d_{1}\right)= \begin{cases}\lambda(A) & \text { se } \theta \in A \\ 0 & \text { se } \theta \notin A\end{cases}
$$

$\mathrm{e}$

$$
l_{A^{c}}\left(\theta, d_{1}\right)= \begin{cases}\lambda\left(A^{c}\right) & \text { se } \theta \in A^{c} \\ 0 & \text { se } \theta \notin A^{c}\end{cases}
$$

segue que $l_{A}\left(\theta, d_{0}\right)=l_{A^{c}}\left(\theta, d_{1}\right)$.

Portanto, como valem (i) e (ii), então essas funções de perda obedecem à proposição 3.7 .

Exemplo 3.6. Considere, para cada $A \in \beta$, uma função de perda $l_{A}$ para testar $H_{0}: \theta \in A$ contra $H_{1}: \theta \notin A$ dada abaixo

$$
l_{A}\left(\theta, d_{0}\right)=g(\operatorname{dist}(\theta, A))=g(\inf \{d(\theta, x): x \in A\})
$$

$e$

$$
l_{A}\left(\theta, d_{1}\right)=g\left(\operatorname{dist}\left(\theta, A^{c}\right)\right)
$$

onde $d(x, y)$ denota uma distância entre os pontos $x$ e y em $\Theta$ e $g: \Re_{+} \rightarrow \Re$ é uma função não-decrescente. A classe de funções de perda $\left\{l_{A}: A \in \beta\right\}$ obedece à proposição 3.7.

Demonstração. Sejam $A, B \subseteq \beta \operatorname{com} A \subseteq B$. Então $\operatorname{dist}(\theta, A) \geq \operatorname{dist}(\theta, B)$ e, como $g($.$) é não-$ decrescente, $g(\operatorname{dist}(\theta, A)) \geq g(\operatorname{dist}(\theta, B))$. Como $l_{A}\left(\theta, d_{0}\right)=g(\operatorname{dist}(\theta, A))$ e $l_{B}\left(\theta, d_{0}\right)=g(\operatorname{dist}(\theta, B))$, segue que $l_{A}\left(\theta, d_{0}\right) \geq l_{B}\left(\theta, d_{0}\right)$.

Por outro lado $l_{A}\left(\theta, d_{1}\right)=g\left(\operatorname{dist}\left(\theta, A^{c}\right)\right)$, então $l_{A^{c}}\left(\theta, d_{1}\right)=g(\operatorname{dist}(\theta, A))=l_{A}\left(\theta, d_{0}\right)$. Portanto, como valem (i) e (ii), essa classe de funções de perda obedece à proposição 3.7.

Outro grupo de funções de perda que confere monotonicidade a testes simultâneos foi proposto por Lavine e Schervish [10]. Os autores apresentam funções de perda que, uma vez adotadas, fazem com que testes incoerentes (que não apresentam monotonicidade) não sejam decisões de Bayes, no caso Bayesiano, ou que sejam inadmissíveis, no caso clássico.

Adaptando os resultados obtidos por Lavine e Schervish [10], espera-se que uma classe de testes de hipóteses obtida a partir de funções de perda submetidas às condições estabelecidas por eles 
tenha monotonicidade, como será visto a seguir.

Proposição 3.8. Sejam $\chi, \Theta$ e $\beta$ como na definição 3.1. Para cada $A \in \beta$, seja $l_{A}: \Theta \times D=$ $\left\{d_{0}, d_{1}\right\} \rightarrow \Re_{+}$uma função de perda que, para cada par $(\theta, d) \in \Theta \times D$ associa a perda $l_{A}(d, \theta)$ quando se testa $H_{0}: \theta \in A$ contra $H_{1}: \theta \notin A$. Assim, temos uma classe de funções de perda $\left\{l_{A}: A \in \beta\right\}$. Suponha que a classe satisfaça

$$
\begin{gathered}
(i) l_{A}\left(\theta, d_{0}\right) \geq l_{A}\left(\theta, d_{1}\right), \forall \theta \notin A, \\
(i i) l_{A}\left(\theta, d_{0}\right) \leq l_{A}\left(\theta, d_{1}\right), \forall \theta \in A, e \\
(i i i) l_{A}\left(\theta, d_{0}\right)-l_{A}\left(\theta, d_{1}\right)=l_{B}\left(\theta, d_{0}\right)-l_{B}\left(\theta, d_{1}\right), \forall \theta \in(A \cap B) \cup\left(A^{c} \cap B^{c}\right) .
\end{gathered}
$$

Então, a classe de testes que para cada $A \in \beta$ associa o teste de Bayes para testar $H_{0}: \theta \in A$ contra $H_{1}: \theta \notin A$ obtido considerando a função de perda $l_{A}$ tem monotonicidade.

Demonstração. Sejam $A, B \in \beta \operatorname{com} A \subseteq B$. Seja $x \in \chi$ tal que $\tau_{A}(x)=0$. Seja $P_{x}$ a distribuição de probabilidade de $\theta$ dado $x$. Assim, $E\left[l_{A}\left(\theta, d_{0}\right) / x\right]<E\left[l_{A}\left(\theta, d_{1}\right) / x\right]$, então

$$
\begin{gathered}
\int_{A} l_{A}\left(\theta, d_{0}\right) d P_{x}+\int_{B \cap A^{c}} l_{A}\left(\theta, d_{0}\right) d P_{x}+\int_{B^{c} \cap A^{c}} l_{A}\left(\theta, d_{0}\right) d P_{x}< \\
<\int_{A} l_{A}\left(\theta, d_{1}\right) d P_{x}+\int_{B \cap A^{c}} l_{A}\left(\theta, d_{1}\right) d P_{x}+\int_{B^{c} \cap A^{c}} l_{A}\left(\theta, d_{1}\right) d P_{x} \Rightarrow \\
\Rightarrow \int_{A}\left[l_{A}\left(\theta, d_{0}\right)-l_{A}\left(\theta, d_{1}\right)\right] d P_{x}+\int_{B \cap A^{c}}\left[l_{A}\left(\theta, d_{0}\right)-l_{A}\left(\theta, d_{1}\right)\right] d P_{x}+ \\
+\int_{B^{c} \cap A^{c}}\left[l_{A}\left(\theta, d_{0}\right)-l_{A}\left(\theta, d_{1}\right)\right] d P_{x}<0 .
\end{gathered}
$$

Por outro lado,

$$
\begin{gathered}
E\left[l_{B}\left(\theta, d_{0}\right) / x\right]-E\left[l_{B}\left(\theta, d_{1}\right) / x\right]= \\
=\int_{A}\left[l_{A}\left(\theta, d_{0}\right)-l_{A}\left(\theta, d_{1}\right)\right] d P_{x}+\int_{B \cap A^{c}}\left[l_{B}\left(\theta, d_{0}\right)-l_{B}\left(\theta, d_{1}\right)\right] d P_{x}+ \\
+\int_{B^{c} \cap A^{c}}\left[l_{A}\left(\theta, d_{0}\right)-l_{A}\left(\theta, d_{1}\right)\right] d P_{x},
\end{gathered}
$$

onde a última igualdade decorre de (iii). Além disso, pela propriedade (i),

$$
\int_{B \cap A^{c}}\left[l_{A}\left(\theta, d_{0}\right)-l_{A}\left(\theta, d_{1}\right)\right] d P_{x} \geq 0
$$

e, pela propriedade (ii),

$$
\int_{B \cap A^{c}}\left[l_{B}\left(\theta, d_{0}\right)-l_{B}\left(\theta, d_{1}\right)\right] d P_{x} \leq 0 .
$$


Juntando estes fatos com 3.7, temos que

$$
\begin{gathered}
E\left[l_{B}\left(\theta, d_{0}\right) / x\right]-E\left[l_{B}\left(\theta, d_{1}\right) / x\right]=\int_{A}\left[l_{B}\left(\theta, d_{0}\right)-l_{B}\left(\theta, d_{1}\right)\right] d P_{x}+ \\
+\int_{B \cap A^{c}}\left[l_{B}\left(\theta, d_{0}\right)-l_{B}\left(\theta, d_{1}\right)\right] d P_{x}+\int_{B^{c} \cap A^{c}}\left[l_{B}\left(\theta, d_{0}\right)-l_{B}\left(\theta, d_{1}\right)\right] d P_{x} \leq \\
\leq \int_{A}\left[l_{A}\left(\theta, d_{0}\right)-l_{A}\left(\theta, d_{1}\right)\right] d P_{x}+ \\
+\int_{A^{c} \cap B}\left[l_{A}\left(\theta, d_{0}\right)-l_{A}\left(\theta, d_{1}\right)\right] d P_{x}+\int_{A^{c} \cap B^{c}}\left[l_{A}\left(\theta, d_{0}\right)-l_{A}\left(\theta, d_{1}\right)\right] d P_{x}<0 .
\end{gathered}
$$

Ou seja, se a classe de funções de perda obedecer a (i), (ii) e (iii), então $E\left[l_{A}\left(\theta, d_{0}\right) / x\right]<$ $E\left[l_{A}\left(\theta, d_{1}\right) / x\right]$ implica que $E\left[l_{B}\left(\theta, d_{0}\right) / x\right]<E\left[l_{B}\left(\theta, d_{1}\right) / x\right]$ e, portanto, a classe de testes de Bayes apresenta monotonicidade.

As condições $(i)$ e $(i i)$ da classe de funções de perda $\left\{l_{A}: A \in \beta\right\}$ da proposição 3.8 exigem que uma tomada de decisão equivocada seja mais penalizada do que a tomada de uma decisão correta. Por exemplo, na condição $(i)$, a perda por aceitar $H_{0}$ quando $H_{0}$ é falso é maior que a perda por rejeitar $H_{0}$ quando $H_{0}$ é falso, o que é bastante intuitivo.

A condição (iii) exige que, quando $\theta \in(A \cap B)$ ou $\theta \in\left(A^{c} \cap B^{c}\right)$, a diferença entre as perdas por aceitar e rejeitar $H_{0}$ seja igual a diferença entre as perdas por aceitar e rejeitar $H_{0}^{\prime}$, ou seja, quando o verdadeiro valor do parâmetro está tanto em $A$ quanto em $B$ (ou em nenhum deles). Schervish e Lavine [10] sugerem que não há motivo para que as diferenças entre as perdas associadas a acerto e erro sejam diferentes.

Um exemplo de classe de funções de perda que atende a proposição 3.8 é: para $A \in \beta, l_{A}\left(\theta, d_{0}\right)=$ $a I_{A^{c}}(\theta)$ e $l_{A}\left(\theta, d_{1}\right)=b I_{A}(\theta), a, b>0$. É fácil ver que vale (i), pois, para $\theta \in A, l_{A}\left(\theta, d_{0}\right)=0<$ $b=l_{A}\left(\theta, d_{1}\right)$. Vale também (ii), pois, para $\theta \in A^{c}, l_{A}\left(\theta, d_{1}\right)=0<a=l_{A}\left(\theta, d_{0}\right)$. Por fim, vale (iii), pois para $\theta \in(A \cap B), l_{A}\left(\theta, d_{0}\right)-l_{A}\left(\theta, d_{1}\right)=0-b=l_{B}\left(\theta, d_{0}\right)-l_{B}\left(\theta, d_{1}\right)$ (analogamente, verifica-se para o caso $\left.\theta \in A^{c} \cap B^{c}\right)$.

As proposições estudadas neste capítulo apresentam condições suficientes para que classes de testes de hipóteses tenham monotonicidade. Porém, não são estudadas caracterizações das classes de testes com monotonicidade, ou seja, dado que uma classe de testes apresenta monotonicidade, não se sabe caracterizar a correspondente classe de funções de perda. Em outras palavras, não são apresentadas condições necessárias para a monotonicidade. 


\section{Capítulo 4}

\section{Conclusões}

\subsection{Considerações Finais}

Neste trabalho, foi levantado um problema de coerência lógica presente em alguns testes de hipóteses simultâneos muito utilizados na literatura. Um dos primeiros artigos tratando desse problema foi Gabriel [9] que diz que caso uma hipótese testada por um teste de significância não é rejeitada, espera-se que em todos os problemas em que a hipótese nula é implicada pela primeira também não seja rejeitada a hipótese nula, a partir de uma mesma observação $x$. À propriedade que trata dessa coerência lógica dá-se o nome, aqui, de monotonicidade.

Neste trabalho, foi definida formalmente essa propriedade e explicado porque é importante que procedimentos de teste de hipóteses tenham esta propriedade. Também foi introduzido o conceito de classe de testes de hipóteses, uma generalização de um procedimento de testes para uma sigmaálgebra de subconjuntos de hipóteses nulas.

Foram verificadas, para diversas classes de testes conhecidas na literatura, quais apresentam a propriedade de monotonicidade. Além de verificar, quando possível, quais as condições suficientes, sob a Teoria da Decisão, para que uma classe de testes de Bayes tenha esta propriedade.

Para a classe de testes Razão de Verossimilhanças Generalizada, com nível de significância fixado, foi mostrado que, mesmo em exemplos simples, não apresenta a propriedade de monotonicidade. Para a classe de testes de Bayes baseados em probabilidades a posteriori, para a classe de testes FBST e para a classe de testes de Lindley, é mostrado que, fixado um valor para $\alpha \in(0,1)$, elas sempre apresentam monotonicidade.

Sob a abordagem da Teoria da Decisão, foi mostrado que algumas funções de perda intuitivas conferem monotonicidade à classe testes de Bayes baseados em probabilidades a posteriori, além de apresentadas classes de funções de perda que sempre conduzem a classes de testes bayesianos com monotonicidade.

\subsection{Sugestões para Pesquisas Futuras}

- Examinar outros aspectos lógicos relativos aos testes de hipóteses simultâneos.

- Caracterizar a propriedade de monotonicidade, ou seja, encontrar as condições necessárias para que uma classe de testes de hipóteses tenha monotonicidade. 
- Estudar a concordância lógica de testes com duas decisões (testes de hipóteses usuais) com problemas de decisão com mais de duas alternativas.

- Estudar a propriedade de monotonicidade de testes bayesianos considerando funções de perda que dependam do tamanho da amostra e das dimensões das hipóteses.

- Caracterizar monotonicidade sob o ponto de vista de funções de conjuntos (hipóteses nulas). 


\section{Apêndice A}

\section{Demonstrações}

\section{A.1 Construção da Região Crítica do teste RVG para o modelo de ANOVA}

A função de verossimilhança é dada por

$L\left(\mu_{X}, \mu_{Y}, \mu_{Z}, \sigma^{2} / x, y, z\right)=\prod_{i=1}^{n}\left\{\frac{1}{\sqrt{2 \pi} \sigma} \exp \left(\frac{-\left(x_{i}-\mu_{X}\right)^{2}}{2 \sigma^{2}}\right) \frac{1}{\sqrt{2 \pi} \sigma} \exp \left(\frac{-\left(y_{i}-\mu_{Y}\right)^{2}}{2 \sigma^{2}}\right) \frac{1}{\sqrt{2 \pi} \sigma} \exp \left(\frac{-\left(z_{i}-\mu_{Z}\right)^{2}}{2 \sigma^{2}}\right)\right\}$.

Nas condições da subseção 3.3.3, considerando a hipótese $H_{0}: \mu_{X}=\mu_{Y}=\mu_{Z}$. Sob $H_{0}$, a função de verossimilhança é dada por:

$L\left(\mu, \mu, \mu, \sigma^{2} / x, y, z\right)=\prod_{i=1}^{n}\left\{\frac{1}{\sqrt{2 \pi} \sigma} \exp \left(\frac{-\left(x_{i}-\mu\right)^{2}}{2 \sigma^{2}}\right) \frac{1}{\sqrt{2 \pi} \sigma} \exp \left(\frac{-\left(y_{i}-\mu\right)^{2}}{2 \sigma^{2}}\right) \frac{1}{\sqrt{2 \pi} \sigma} \exp \left(\frac{-\left(z_{i}-\mu\right)^{2}}{2 \sigma^{2}}\right)\right\}$.

Calculando $l\left(\mu, \sigma^{2} / x, y, z\right)$, o logaritmo de $L\left(\mu, \mu, \mu, \sigma^{2} / x, y, z\right)$ temos:

$l\left(\mu, \sigma^{2} / x, y, z\right)=\frac{-3 n \log (2 \pi)}{2}+\frac{-3 n \log \left(\sigma^{2}\right)}{2}-\sum_{i=1}^{n} \frac{\left(x_{i}-\mu\right)^{2}}{2 \sigma^{2}}-\sum_{i=1}^{n} \frac{\left(y_{i}-\mu\right)^{2}}{2 \sigma^{2}}-\sum_{i=1}^{n} \frac{\left(z_{i}-\mu\right)^{2}}{2 \sigma^{2}}$.

Derivando em $\mu$ e igualando a zero,

$$
\frac{d l\left(\mu, \sigma^{2} / x, y, z\right)}{d \mu}=\sum_{i=1}^{n} \frac{2\left(x_{i}-\mu\right)}{2 \sigma^{2}}+\sum_{i=1}^{n} \frac{2\left(y_{i}-\mu\right)}{2 \sigma^{2}}+\sum_{i=1}^{n} \frac{2\left(z_{i}-\mu\right)}{2 \sigma^{2}}=0
$$

Assim, o máximo de $l\left(\mu, \sigma^{2} / x, y, z\right)$ sob a hipótese $H_{0}$ é atingido em

$$
\widehat{\mu_{0}}=\frac{\sum_{i=1}^{n} x_{i}+\sum_{i=1}^{n} y_{i}+\sum_{i=1}^{n} z_{i}}{3 n}
$$

independentemente do valor de $\sigma^{2}$. Agora derivando $l\left(\widehat{\mu_{0}}, \sigma^{2} / x, y, z\right)$ em relação a $\sigma^{2}$ e igualando a zero, temos que o máximo de $l\left(\widehat{\mu_{0}}, \sigma^{2} / x, y, z\right)$ é atingido em

$$
\widehat{\sigma_{0}^{2}}=\frac{\sum_{i=1}^{n}\left(x_{i}-\widehat{\mu}_{0}\right)^{2}+\sum_{i=1}^{n}\left(y_{i}-\widehat{\mu}_{0}\right)^{2}+\sum_{i=1}^{n}\left(z_{i}-\widehat{\mu}_{0}\right)^{2}}{3 n} .
$$


Em $\Theta$, o máximo de $L\left(\mu_{X}, \mu_{Y}, \mu_{Z}, \sigma^{2} / x, y, z\right)$ é atingido por

$$
\begin{gathered}
\widehat{\mu}_{X}=\bar{x}, \\
\widehat{\mu}_{Y}=\bar{y}, \\
\widehat{\mu}_{Z}=\bar{z}, \\
\widehat{\sigma}^{2}=\frac{\sum_{i=1}^{n}\left(x_{i}-\widehat{\mu}_{X}\right)^{2}+\sum_{i=1}^{n}\left(y_{i}-\widehat{\mu}_{Y}\right)^{2}+\sum_{i=1}^{n}\left(z_{i}-\widehat{\mu}_{Z}\right)^{2}}{3 n} .
\end{gathered}
$$

Pela definição 2.5, rejeita-se $H_{0}$ se, e somente se, $\lambda(x, y, z) \leq c$. Aqui,

$$
\lambda(x, y, z)=\frac{L\left(\widehat{\mu}_{0}, \widehat{\mu}_{0}, \widehat{\mu}_{0}, \widehat{\sigma}_{0}^{2} / x, y, z\right)}{L\left(\widehat{\mu}_{X}, \widehat{\mu}_{Y}, \widehat{\mu}_{Z}, \widehat{\sigma}^{2} / x, y, z\right)}=\left(\frac{\widehat{\sigma}^{2}}{\widehat{\sigma}_{0}^{2}}\right)^{\frac{3 n}{2}} .
$$

Utilizando a notação tradicional para Análise de Variância, onde

$$
\begin{gathered}
S Q T=\sum_{i=1}^{n}\left(x_{i}-\widehat{\mu}_{0}\right)^{2}+\sum_{i=1}^{n}\left(y_{i}-\widehat{\mu}_{0}\right)^{2}+\sum_{i=1}^{n}\left(z_{i}-\widehat{\mu}_{0}\right)^{2}, \\
S Q R=\sum_{i=1}^{n}\left(x_{i}-\widehat{\mu}_{X}\right)^{2}+\sum_{i=1}^{n}\left(y_{i}-\widehat{\mu}_{Y}\right)^{2}+\sum_{i=1}^{n}\left(z_{i}-\widehat{\mu}_{Z}\right)^{2}, \\
S Q E=\sum_{i=1}^{n}\left(\widehat{\mu}_{X}-\widehat{\mu}_{0}\right)^{2}+\sum_{i=1}^{n}\left(\widehat{\mu}_{Y}-\widehat{\mu}_{0}\right)^{2}+\sum_{i=1}^{n}\left(\widehat{\mu}_{Z}-\widehat{\mu}_{0}\right)^{2}, \\
Q M T=\frac{S Q T}{3 n-1}, \\
Q M R=\frac{S Q R}{3 n-3} \mathrm{e} \\
Q M E=\frac{S Q E}{2},
\end{gathered}
$$

de modo que $S Q T=S Q R+S Q E$.

Ou seja, rejeita-se $H_{0}$ se, e somente se,

$$
\begin{aligned}
\left(\frac{\widehat{\sigma}^{2}}{\widehat{\sigma}_{0}^{2}}\right)^{\frac{3 n}{2}} \leq c & \Leftrightarrow\left(\frac{S Q R}{S Q T}\right)^{\frac{3 n}{2}} \leq c \\
& \Leftrightarrow \frac{S Q R}{S Q R+S Q E} \leq c^{\prime} \\
& \Leftrightarrow \frac{S Q R+S Q E}{S Q R} \geq \frac{1}{c^{\prime}} \\
& \Leftrightarrow 1+\frac{S Q E}{S Q R} \geq \frac{1}{c^{\prime}} \\
& \Leftrightarrow \frac{S Q E}{S Q R} \geq c^{\prime \prime}
\end{aligned}
$$




$$
\Leftrightarrow \frac{Q M E}{Q M R} \geq k
$$

onde $k$ é dado por $\alpha=\sup _{\theta \in \Theta_{0}} P\left(\frac{Q M E}{Q M R} \geq k / \theta\right)$.

Seja $\alpha=0,05$. Sob $H_{0}, \frac{Q M E}{Q M R} \sim F_{2,30}$ (Neter et all [15]). Para $\theta \in A$, o valor de $k$ tal que $P\left(\frac{Q M E}{Q M R} \geq k / \theta\right)=0,05$ é $k=3,32$.

Portanto, a região crítica do teste RVG para este modelo de Análise de Variância é

$$
R C=\left\{(x, y, z) \in \chi: \frac{Q M E}{Q M R} \geq 3,32\right\}
$$

\section{A.2 Região crítica do teste RVG para comparação entre médias de dois grupos}

A função de verossimilhança é dada por

$L\left(\mu_{X}, \mu_{Y}, \mu_{Z}, \sigma^{2} / x, y, z\right)=\prod_{i=1}^{n}\left\{\frac{1}{\sqrt{2 \pi} \sigma} \exp \left(\frac{-\left(x_{i}-\mu_{X}\right)^{2}}{2 \sigma^{2}}\right) \frac{1}{\sqrt{2 \pi} \sigma} \exp \left(\frac{-\left(y_{i}-\mu_{Y}\right)^{2}}{2 \sigma^{2}}\right) \frac{1}{\sqrt{2 \pi} \sigma} \exp \left(\frac{-\left(z_{i}-\mu_{Z}\right)^{2}}{2 \sigma^{2}}\right)\right\}$.

Nas condições da subseção 3.3.3, considerando a hipótese $H_{0}^{\prime}: \mu_{X}=\mu_{Z}$, a função de verossimilhança é dada por:

$L\left(\mu, \mu_{Y}, \mu, \sigma^{2} / x, y, z\right)=\prod_{i=1}^{n}\left\{\frac{1}{\sqrt{2 \pi} \sigma} \exp \left(\frac{-\left(x_{i}-\mu\right)^{2}}{2 \sigma^{2}}\right) \frac{1}{\sqrt{2 \pi} \sigma} \exp \left(\frac{-\left(y_{i}-\mu_{Y}\right)^{2}}{2 \sigma^{2}}\right) \frac{1}{\sqrt{2 \pi} \sigma} \exp \left(\frac{-\left(z_{i}-\mu\right)^{2}}{2 \sigma^{2}}\right)\right\}$.

Calculando $l\left(\mu, \mu_{Y}, \sigma^{2} / x, y, z\right)$, o logaritmo de $L\left(\mu, \mu_{Y}, \mu, \sigma^{2} / x, y, z\right)$,

$l\left(\mu, \mu_{Y}, \sigma^{2} / x, y, z\right)=\frac{-3 n \log (2 \pi)}{2}+\frac{-3 n \log \left(\sigma^{2}\right)}{2}-\sum_{i=1}^{n} \frac{\left(x_{i}-\mu\right)^{2}}{2 \sigma^{2}}-\sum_{i=1}^{n} \frac{\left(y_{i}-\mu_{Y}\right)^{2}}{2 \sigma^{2}}-\sum_{i=1}^{n} \frac{\left(z_{i}-\mu\right)^{2}}{2 \sigma^{2}}$.

Derivando em $\mu$ e igualando a zero temos,

$$
\frac{\left.d l\left(\mu, \mu_{Y}, \sigma^{2} / x, y, z\right)\right)}{d \mu}=\frac{\sum_{i=1}^{n} 2\left(x_{i}-\mu\right)}{2 \sigma^{2}}+\frac{\sum_{i=1}^{n} 2\left(z_{i}-\mu\right)}{2 \sigma^{2}}=0 .
$$

Assim, o máximo de $l\left(\mu, \mu_{Y}, \sigma^{2} / x, y, z\right)$ sob a hipótese $H_{0}^{\prime}$ é atingido em

$$
\begin{gathered}
\widehat{\mu_{0}^{\prime}}=\frac{\sum_{i=1}^{n} x_{i}+\sum_{i=1}^{n} z_{i}}{2 n} \mathrm{e} \\
\widehat{\mu_{Y}}=\bar{y}
\end{gathered}
$$

independentemente do valor de $\sigma^{2}$. Agora derivando $l\left(\mu, \mu_{Y}, \sigma^{2} / x, y, z\right)$ em relação a $\sigma^{2}$ e igualando 
a zero, temos que o máximo de $l\left(\mu, \mu_{Y}, \sigma^{2} / x, y, z\right)$ é atingido em

$$
\widehat{\sigma_{0}^{2 \prime}}=\frac{\sum_{i=1}^{n}\left(x_{i}-\widehat{\mu}_{0}^{\prime}\right)^{2}+\sum_{i=1}^{n}\left(y_{i}-\overline{\mu_{Y}}\right)^{2}+\sum_{i=1}^{n}\left(z_{i}-\widehat{\mu}_{0}^{\prime}\right)^{2}}{3 n} .
$$

Em $\Theta$, o máximo de $L\left(\mu_{X}, \mu_{Y}, \mu_{Z}, \sigma^{2}\right)$ é atingido por

$$
\begin{gathered}
\widehat{\mu}_{X}=\bar{x}, \\
\widehat{\mu}_{Y}=\bar{y}, \\
\widehat{\mu}_{Z}=\bar{z}, \\
\widehat{\sigma}^{2}=\frac{\sum_{i=1}^{n}\left(x_{i}-\widehat{\mu}_{X}\right)^{2}+\sum_{i=1}^{n}\left(y_{i}-\widehat{\mu}_{Y}\right)^{2}+\sum_{i=1}^{n}\left(z_{i}-\widehat{\mu}_{Z}\right)^{2}}{3 n} .
\end{gathered}
$$

Pela definição 2.5, rejeita-se $H_{0}$ se, e somente se, $\lambda(x, y, z) \leq c$. Aqui,

$$
\lambda(x, y, z)=\frac{L\left(\widehat{\mu}_{0}^{\prime}, \widehat{\mu}_{Y}^{\prime}, \widehat{\mu}_{0}^{\prime}, \widehat{\sigma}^{2 \prime} / x, y, z\right)}{L\left(\widehat{\mu}_{X}, \widehat{\mu}_{Y}, \widehat{\mu}_{Z}, \widehat{\sigma}^{2}, / x, y, z\right)}=\left(\frac{\widehat{\sigma}^{2}}{\widehat{\sigma}_{0}^{2 \prime}}\right)^{\frac{3 n}{2}} .
$$

Ou seja, rejeita-se $H_{0}^{\prime}$ se, e somente se,

$$
\left(\frac{\widehat{\sigma}^{2}}{\widehat{\sigma}_{0}^{2 \prime}}\right)^{\frac{3 n}{2}} \leq c \Longleftrightarrow\left(\frac{\sum_{i=1}^{n}\left(x_{i}-\bar{x}\right)^{2}+\sum_{i=1}^{n}\left(y_{i}-\bar{y}\right)^{2}+\sum_{i=1}^{n}\left(z_{i}-\bar{z}\right)^{2}}{\sum_{i=1}^{n}\left(x_{i}-\widehat{\mu_{0}^{\prime}}\right)^{2}+\sum_{i=1}^{n}\left(y_{i}-\bar{y}\right)^{2}+\sum_{i=1}^{n}\left(z_{i}-\widehat{\mu_{0}^{\prime}}\right)^{2}}\right)^{\frac{3 n}{2}} \leq c .
$$

Sabe-se que $\sum_{i=1}^{n}\left(x_{i}-\widehat{\mu_{0}^{\prime}}\right)^{2}=\sum_{i=1}^{n}\left(x_{i}-\bar{x}\right)^{2}+n\left(\bar{x}-\widehat{\mu_{0}^{\prime}}\right)^{2}$. Dessa forma,

$$
\begin{gathered}
\left(\frac{\widehat{\sigma}^{2}}{\widehat{\sigma}_{0}^{2 \prime}}\right)^{\frac{3 n}{2}} \leq c \Leftrightarrow \frac{1}{\frac{\sum_{i=1}^{n}\left(x_{i}-\bar{x}\right)^{2}+n\left(\bar{x}-\widehat{\mu_{0}^{\prime}}\right)^{2}+\sum_{i=1}^{n}\left(y_{i}-\bar{y}\right)^{2}+\sum_{i=1}^{n}\left(z_{i}-\bar{z}\right)^{2}+n\left(\bar{z}-\widehat{\mu_{0}^{\prime}}\right)^{2}}{\sum_{i=1}^{n}\left(x_{i}-\bar{x}\right)^{2}+\sum_{i=1}^{n}\left(y_{i}-\bar{y}\right)^{2}+\sum_{i=1}^{n}\left(z_{i}-\bar{z}\right)^{2}}} \leq c \Leftrightarrow \\
\Longleftrightarrow \frac{1}{1+\frac{n\left(\bar{x}-\widehat{\mu_{0}^{\prime}}\right)^{2}+n\left(\bar{z}-\widehat{\mu_{0}^{\prime}}\right)^{2}}{\sum_{i=1}^{n}\left(x_{i}-\bar{x}\right)^{2}+\sum_{i=1}^{n}\left(y_{i}-\bar{y}\right)^{2}+\sum_{i=1}^{n}\left(z_{i}-\bar{z}\right)^{2}}} \leq c \Leftrightarrow \\
\Longleftrightarrow \frac{n\left(\bar{x}-\hat{\mu_{0}^{\prime}}\right)^{2}+n\left(\bar{z}-\hat{\mu_{0}^{\prime}}\right)^{2}}{Q M R} \geq c_{1} .
\end{gathered}
$$

Como $\widehat{\mu_{0}^{\prime}}=\frac{\bar{x}+\bar{z}}{2}$, então $\bar{x}-\widehat{\mu_{0}^{\prime}}=\frac{1}{2}(\bar{x}-\bar{z})$ e $\bar{z}-\widehat{\mu_{0}^{\prime}}=\frac{1}{2}(\bar{z}-\bar{x})$ e, portanto,

$$
\left(\frac{\widehat{\sigma}^{2}}{\widehat{\sigma}_{0}^{2 \prime}}\right)^{\frac{3 n}{2}} \leq c \Leftrightarrow \frac{\frac{n}{4}(\bar{x}-\bar{z})^{2}+\frac{n}{4}(\bar{z}-\bar{x})^{2}}{Q M R} \geq c_{1} \Longleftrightarrow \frac{\frac{n}{2}(\bar{x}-\bar{z})^{2}}{Q M R} \geq c_{1} .
$$


Portanto, rejeita-se $H_{0}^{\prime}$ se, e somente se,

$$
\left|\frac{\bar{x}-\bar{z}}{\sqrt{Q M R} \sqrt{\frac{2}{n}}}\right| \geq k_{1}
$$

Seja $\alpha=0,05$. Sob $H_{0}^{\prime}, \frac{\bar{X}-\bar{Z}}{\sqrt{Q M R} \sqrt{\frac{2}{n}}} \sim t_{30}$ (Neter et all [15]). Para $\theta \in B$, o valor de $k_{1}$ tal que $P\left(\left|\frac{\bar{X}-\bar{Z}}{\sqrt{Q M R} \sqrt{\frac{2}{n}}}\right|>k_{1} / \theta\right)=0,05$ é $k_{1}=2,042$.

Portanto, a região crítica do teste RVG para comparação de duas médias, neste caso, é

$$
R C^{\prime}=\left\{(x, y, z) \in \chi:\left|\frac{\bar{x}-\bar{z}}{\sqrt{Q M R} \sqrt{\frac{2}{n}}}\right|>2,042\right\}
$$




\section{Referências Bibliográficas}

[1] J.O. Berger and M. Delampady, Testing precise hypotheses, Statistical Science 2 (1987), 317352. 8

[2] J.M. Bernardo and A.F.M. Smith, Bayesian theory, John Wiley and Sons, Chicester, 2004. 10

[3] M.A. Betz and J.R. Levin, Coherent analysis-of-variance hypothesis-testing strategies: A general model, Journal of Educational Statistics 7 (1982), 193-206. 1

[4] H. Bolfarine and M.C. Sandoval, Introdução à inferência estatística, first ed., Sociedade Brasileira de Matemática, Rio de Janeiro, 2001. 5

[5] G. Casella and R.L. Berger, Statistical inference, second ed., Duxbury Advanced Series, California, 2002. 3

[6] M. DeGroot, Probability and statistics, second ed., Addilson-Wesley Publishing Company, Massachusetts, 1986. 4, 5

[7] _ Optimal decision, fourth ed., Garland Science, New York, 2002. 6

[8] V. Fossaluza, Testes de hipóteses em eleições majoritárias, 2008. 2, 16, 17

[9] K.R. Gabriel, Simultaneus test procedures - some theory of multiple comparisons, The Annals of Mathematical Statistics 40 (1969), 224-250. 1, 2, 15, 31

[10] M. Lavine and M.J. Schervish, Bayes factors: What they are and what they are not, The American Statistician 53 (1999), 119-122. 2, 28, 30

[11] E.L. Lehmann, A theory of some multiple decision problems i, The Annals of Mathematical Statistics 28 (1957), 1-25. 1

[12] E.L. Lehmann and J.P. Romano, Testing statistical hypoteses, third ed., Springer Texts in Statistics, New York, 2005. 3, 5

[13] D.V. Lindley, Introduction to probability and statistics: from a bayesian viewpoint part 2, Cambridge University Press, London, 1965. 9, 10

[14] M.R. Madruga, L.G. Esteves, and S. Wechsler, On the bayesianity of pereira-stern tests, Sociedad de Estadística e Investigacíon Operativa Test 10 (2001), 291-299. 8

[15] J. Neter, M. H. Kutner, C. J. Nachtsheim, and W. Li, Applied linear statistical models, fifht ed., McGraw-Hill, New York, 2004. 21, 35, 37

[16] C.A.B. Pereira and J.M. Stern, Evidence and credibility: Full bayesian significance test for precise hypoteses, Entropy 1 (1999), 104-115. 3, 8 
[17] C.A.B. Pereira, J.M. Stern, and S. Wechsler, Can a significance test be genuinely bayesian?, Bayesian Analysis 3 (2008), 79-100. 8

[18] D.M. Rom and B. Holland, A new closed multiple testing procedure for hierarchical families of hypotheses, Journal of Statistical Planning and Inference 46 (1995), 265-275. 1

[19] M.J. Schervish, $P$ values: What they are and what they are not, The American Statistician 50 (1996), 203-206. 1, 15

[20] J.M. Stern, Cognitive constructivism and the epistemic significance of sharp statistical hypotheses, 28th International Workshop on Bayesian Inference and Maximum Entropy Mehtods in Science and Enginnering, São Paulo, 2008. 8

[21] A. Zellner, An introduction to bayesian inference in econometrics, Wiley series in probability and mathematical statistics, New York, 1971. 9, 11 